\title{
On approximate solutions of semilinear evolution equations.
}

\author{
${\text { Carlo } \text { Morosi }^{1} \text {, Livio Pizzocchero }}^{2}$ \\ ${ }^{1}$ Dipartimento di Matematica, Politecnico di Milano, \\ P.za L. da Vinci 32, I-20133 Milano, Italy \\ e-mail: carmor@mate.polimi.it \\ 2 Dipartimento di Matematica, Università di Milano \\ Via C. Saldini 50, I-20133 Milano, Italy \\ and Istituto Nazionale di Fisica Nucleare, Sezione di Milano, Italy \\ e-mail: livio.pizzocchero@mat.unimi.it
}

\begin{abstract}
A general framework is presented to discuss the approximate solutions of an evolution equation in a Banach space, with a linear part generating a semigroup and a sufficiently smooth nonlinear part. A theorem is presented, allowing to infer from an approximate solution the existence of an exact solution. According to this theorem, the interval of existence of the exact solution and the distance of the latter from the approximate solution can be evaluated solving a one-dimensional "control" integral equation, where the unknown gives a bound on the previous distance as a function of time. For example, the control equation can be applied to the approximation methods based on the reduction of the evolution equation to finite-dimensional manifolds: among them, the Galerkin method is discussed in detail. To illustrate this framework, the nonlinear heat equation is considered. In this case the control equation is used to evaluate the error of the Galerkin approximation; depending on the initial datum, this approach either grants global existence of the solution or gives fairly accurate bounds on the blow up time.
\end{abstract}

Keywords: Differential equations, theoretical approximation, nonlinear heat equation, blow up.

AMS 2000 Subject classifications: 34AXX, 35AXX, 35KXX.

\section{To appear in "Reviews in Mathematical Physics".}




\section{Introduction.}

In this paper we consider, within a Banach space $\mathbf{F}$, a Volterra integral equation

$$
\varphi(t)=\mathcal{U}\left(t-t_{0}\right) f_{0}+\int_{t_{0}}^{t} d s \mathcal{U}(t-s) \mathcal{P}(\varphi(s), s),
$$

for an unknown function $\varphi$ from a real interval to $\mathbf{F}$. Here $f_{0} \in \mathbf{F}, \mathcal{U}$ is a linear semigroup on $\mathbf{F}$ and $\mathcal{P}$ is a locally Lipschitz nonlinear map from an open set of $\mathbf{F} \times \mathbf{R}$ to $\mathbf{F}$. If $\mathcal{U}$ is the semigroup generated by a linear operator $\mathcal{A}: \operatorname{Dom} \mathcal{A} \subset \mathbf{F} \rightarrow \mathbf{F}$, under minimal technical conditions the above Volterra equation is equivalent to a Cauchy problem

$$
\dot{\varphi}(t)=\mathcal{A} \varphi(t)+\mathcal{P}(\varphi(t), t), \quad \varphi\left(t_{0}\right)=f_{0}, \quad(\cdot:=d / d t) .
$$

To standardize the language, problems (1.1), (1.2) are defined precisely in Sect 2 local existence and uniqueness of their solutions are well known.

The aim of this paper is to discuss the approximate solutions of (1.1). In the most general sense, an approximate solution is simply a continuous map $t \mapsto \varphi_{a p}(t)$ which can be inserted in the r.h.s. of (1.1), i.e., such that graph $\varphi_{a p} \subset D o m \mathcal{P}$. For any such map, we can define the integral error as the difference between the two sides of (1.1). If $\varphi_{a p}$ is a bit more regular, the integral error is determined by the differential and datum errors which are, respectively, the differences between the two sides in the differential equation and in the initial condition of (1.2).

All the above concepts are formalized in Sect 3. Here, we also present a general statement (Prop 3.4) which can be applied to an approximate solution $t \mapsto \varphi_{a p}(t)$ to infer the existence of an exact solution $\varphi$ on an appropriate time interval, and also to estimate the difference $\varphi(t)-\varphi_{a p}(t)$. The essential character in Prop 3.4 is an integral control inequality, depending on the available estimators for the integral error of $\varphi_{a p}$ and for the growth of $\mathcal{P}$ away from the graph of $\varphi_{a p}$.

The unknown in the control inequality is a real, nonnegative function $t \mapsto R(t)$; if a solution $R$ is found to exist on a time interval $\left[t_{0}, t_{1} \mid\right.$ (i.e., either $\left[t_{0}, t_{1}\right]$ or $\left[t_{0}, t_{1}\right)$ ), then it is granted that (1.1) possesses an exact solution $\varphi:\left[t_{0}, t_{1} \mid \rightarrow \mathbf{F}\right.$, and that $\left\|\varphi(t)-\varphi_{a p}(t)\right\| \leq R(t)$.

In typical cases, a solution of the previous integral inequality can be constructed solving an ordinary differential equation for $R$, that we call as well the control equation. In this way, the problem of giving estimates on the existence time for (1.1) and on its exact solution $\varphi$, living in $\mathbf{F}$ which is typically of infinite dimension, is reduced to the analysis of a one-dimensional ODE.

Prop 3.4 can be regarded as a general formulation of many statements about specific evolutionary problems, often encountered in the literature. From this viewpoint, the content of this Proposition is not at all surprising: however, the technique we use to prove it is essentially different from the arguments often employed in related situations. The standard way of thinking would suggest to prove Prop 3.4 in two steps: a) derive (via some nonlinear Gronwall lemma [16]) an a priori bound $\left\|\varphi(t)-\varphi_{a p}(t)\right\| \leq R(t)$, holding until $\varphi(t)$ exists; b) show that nonexistence of $\varphi$ on the whole interval $\left[t_{0}, t_{1} \mid\right.$ would contradict the previous bound: this argument is called the "continuation principle" in [19]. 
On the contrary, the proof we propose (in Sect 4) is very direct, and shows that $\varphi$ can be constructed on the whole $\left[t_{0}, t_{1} \mid\right.$ by a convergent Peano-Picard iteration, applying repeatedly the Volterra integral operator to the approximate solution $\varphi_{a p}$. The control inequality ensures the invariance under the Volterra operator of the space of functions with distance $\leq R(t)$ from $\varphi_{a p}(t)$ on $\left[t_{0}, t_{1} \mid\right.$; the confinement to this domain of all iterates of $\varphi_{a p}$, and the local Lispchitz nature of $\mathcal{P}$, allow to prove their convergence to a function $\varphi$, also distant less than $R$ from $\varphi_{a p}$.

As a first, very simple illustration of Prop 3.4 in Sect 5 we apply the control equation to the approximate solution $\varphi_{a p}(t):=0$. In spite of the trivial choice for $\varphi_{a p}$, the control equation gives useful information on the interval of existence and on the growth of the exact solution $\varphi$, depending on the norm $\left\|f_{0}\right\|$ of the initial datum. The accuracy of these predictions is tested on an example, concerning the (one-dimensional) wave equation with polynomial nonlinearity.

A second, more refined application of the control equation is proposed in Sect 6 for the Galerkin scheme (and similar approaches). In the conventional formulation, the Galerkin method is an algorithm to construct approximate solutions $t \mapsto \varphi_{a p}(t)$ of (1.1) with values in a finite-dimensional submanifold of $\mathbf{F}$. In this Section, the standard evolution equations for the coordinates of $\varphi_{a p}(t)$ in the Galerkin submanifold are coupled with the control equation for $R(t)$; in this way, a finite-dimensional system of ODE's gives simultaneously the Galerkin approximate solution $\varphi_{a p}$, an interval $\left[t_{0}, t_{1} \mid\right.$ on which the exact solution $\varphi$ of (1.1) is granted to exist and an upper bound for $\left\|\varphi_{a p}(t)-\varphi(t)\right\|$ on this interval.

In Sect [7] all the previous results are applied to a nonlinear heat equation, working for simplicity in one space dimension (with a spatial coordinate $x \in(0, \pi)$ ). In this case, the Cauchy problem (1.2) (with initial time $t_{0}:=0$ ) is, symbolically,

$$
\dot{\varphi}(x, t)=\varphi_{x x}(x, t)+\varphi(x, t)^{p}, \quad \varphi(x, 0)=f_{0}(x)
$$

with $p \in\{2,3,4, \ldots\}$, to be discussed in the Sobolev space $\mathbf{F}:=H_{0}^{1}(0, \pi)$. The implementation of the general framework in the present case with polynomial nonlinearity requires accurate information on the pointwise product of functions in $H_{0}^{1}(0, \pi)$; in particular, precise estimates are needed for the norm $\|f h\|$ when $f, h$ are in this space (see the Appendix $\AA$ about this, and 11] for more general information about multiplication in Sobolev spaces). To exemplify some general facts about (1.3), in the same Section we consider the initial datum $f_{0}(x):=\sqrt{2 / \pi} A \sin x$. If the (nonnegative) constant $A$ is below a critical value, the control equation for the zero approximate solution suffices to prove existence of a globally defined solution $\varphi:[0,+\infty) \rightarrow \mathbf{F}$ of (1.3). For larger $A$, the same control equation gives a finite lower bound for the existence time of the solution $\varphi$. These conclusions are complementary to the ones arising from a known "blow up" theorem of Kaplan for the nonlinear heat equation (see [5]; a review is given in the Appendix (B). When Kaplan' $\mathrm{s}$ theorem is applied to (1.3) with the previous datum, for sufficiently large $A$ it predicts a finite, explicitly determined upper bound on the existence time of the solution.

Again in Sect 7. we add to the above facts the information arising from application of the control equation to the Galerkin scheme; the chosen Galerkin submanifold is the linear span of finitely many elements in the Fourier basis. As an example, we consider the Galerkin differential equations for two modes, coupled with the control equation for $R$, 
with $p=2$ and the previous $f_{0}$. This system in three unknown real functions can be easily treated by any package for the numerical solution of ODE's; the results obtained by the MATHEMATICA package, for several values of $A$, are presented with some detail. Among other things, the Galerkin approach with the control equation allows to increase the critical value of $A$ below which global existence is granted for (1.3); for $A$ above the new critical value, a better lower bound for the existence time is derived. If $A$ is fairly large, the new lower bound is close to the Kaplan upper bound, which yields an uncertainty between $20 \%$ and $30 \%$ on the existence time of the exact solution. Also, the upper bound $R(t)$ on $\left\|\varphi(t)-\varphi_{a p}(t)\right\|$ is fairly small in comparison with $\left\|\varphi_{a p}(t)\right\|$ for non large $t$.

To some extent, it is surprising that a fairly good accuracy can be obtained combining the control equation with a Galerkin scheme in two modes only. These outcomes encourage us to hope that the same method would give nontrivial information on the Cauchy problem for the equations of fluid dynamics, whose Galerkin approximations in few modes give rise, among others, to the widely studied Lorentz model [9] [15].

\section{Preliminaries.}

Throughout the paper, $\mathbf{F}$ denotes a real or complex Banach space with norm \|\| and elements $f, f_{0}, f_{1}, h, \ldots$. We write $\mathbf{B}\left(f_{0}, \rho\right)$ for the open ball in $\mathbf{F}$ of center $f_{0}$ and radius $\rho$ (if $\rho=+\infty$, this means the whole $\mathbf{F})$. Let us be given a linear operator

$$
\mathcal{A}: \operatorname{Dom\mathcal {A}} \subset \mathbf{F} \rightarrow \mathbf{F}
$$

with domain a linear subspace of $\mathbf{F}$; whenever we speak of a continuous map from/to $\operatorname{Dom} \mathcal{A}$, we always refer to the topology of the graph norm $\|f\|_{\mathcal{A}}:=\|f\|+\|A f\|$ (as well known, Dom $\mathcal{A}$ is complete in this norm if and only if $\mathcal{A}$ is closed). We denote with $\mathcal{L}(\mathbf{F})$ the Banach space of bounded linear operators of (the whole) $\mathbf{F}$ into itself.

We always write $\left[t_{0}, t_{1} \mid\right.$ for a real interval of the form $\left[t_{0}, t_{1}\right]$ or $\left[t_{0}, t_{1}\right.$ ) (always intending $t_{0}<t_{1}$; in the second case, $t_{1}$ can be $\left.+\infty\right)$. If $\psi:\left[t_{0}, t_{1} \mid \rightarrow \mathbf{F}\right.$, the graph of this function and the tube around $\psi$ of any radius $\rho \in(0,+\infty]$ are

$$
\begin{gathered}
\text { graph } \psi:=\left\{(\psi(t), t) \mid t \in\left[t_{0}, t_{1} \mid\right\} \subset \mathbf{F} \times \mathbf{R} .\right. \\
\mathbf{T}(\psi, \rho):=\left\{(f, t) \in \mathbf{F} \times\left[t_{0}, t_{1}||\|f-\psi(t)\|<\rho\right\}\right.
\end{gathered}
$$

(the latter is the whole $\mathbf{F} \times\left[t_{0}, t_{1} \mid\right.$, if $\rho=+\infty$; it becomes $\mathbf{B}\left(f_{0}, \rho\right) \times\left[t_{0}, t_{1} \mid\right.$, if $\psi(t)=$ const. $\left.=f_{0}\right)$.

Linear semigroups on $\mathbf{F}$. This name indicates maps $\mathcal{U}$ such that

$$
\mathcal{U}:[0,+\infty) \rightarrow \mathcal{L}(\mathbf{F}), \quad \mathcal{U}(t+s)=\mathcal{U}(t) \mathcal{U}(s), \quad \mathcal{U}(0)=\mathbf{1}_{\mathbf{F}}
$$

The generator of a linear semigroup $\mathcal{U}$ is the linear operator

$$
\begin{gathered}
A: D o m \mathcal{A} \subset \mathbf{F} \rightarrow \mathbf{F}, \quad f \mapsto \mathcal{A} f \\
D o m \mathcal{A}:=\left\{f \in \mathbf{F}\left|\frac{d}{d t}\right|_{t=0}[\mathcal{U}(t) f] \text { exists }\right\}, \quad \mathcal{A} f:=\left.\frac{d}{d t}\right|_{t=0}[\mathcal{U}(t) f]
\end{gathered}
$$


(with $(d / d t)_{t=0}$ denoting the right derivative).

A linear semigroup $\mathcal{U}$ in $\mathbf{F}$ is strongly continuous if for all $f \in \mathbf{F}$ the map $[0,+\infty) \rightarrow \mathbf{F}$, $t \mapsto \mathcal{U}(t) f$ is continuous. In this case (see, e.g., 2] [19]), the map $(f, t) \mapsto \mathcal{U}(t) f$ is jointly continuous, the generator $\mathcal{A}$ is densely defined in $\mathbf{F}$ and closed, and a) b) hold:

a) $\mathcal{A}$ determines $\mathcal{U}$. For all $f_{0} \in D o m \mathcal{A}$, the function $t \mapsto \mathcal{U}(t) f_{0}$ is the unique function $\varphi$ such that

$$
\varphi \in C([0,+\infty), D o m \mathcal{A}) \cap C^{1}([0,+\infty), \mathbf{F}), \quad \dot{\varphi}(t)=\mathcal{A} \varphi(t) \text { for all } t, \quad \varphi(0)=f_{0} ;
$$

b) for any function $\psi \in C\left(\left[t_{0}, t_{1} \mid, D o m \mathcal{A}\right) \cap C^{1}\left(\left[t_{0}, t_{1} \mid, \mathbf{F}\right)\right.\right.$ and $t$ in this interval, it is

$$
\psi(t)=\mathcal{U}\left(t-t_{0}\right) \psi\left(t_{0}\right)+\int_{t_{0}}^{t} d s \mathcal{U}(t-s)[\dot{\psi}(s)-\mathcal{A} \psi(s)]
$$

(here and in the sequel, the dot indicates the derivative). A linear semigroup $\mathcal{U}$ is uniformly continuous if the map $\mathcal{U}$ is continuous from $[0,+\infty)$ to $\mathcal{L}(\mathbf{F})$ with the standard operator norm (uniformly continuous semigroup); this happens if and only if $\mathcal{U}$ has generator $\mathcal{A} \in$ $\mathcal{L}(\mathcal{F})$, and gives a trivial example of strongly continuous semigroup (extendable to $t<0$ ).

2.1 Definition. An estimator for a linear semigroup $\mathcal{U}$ on $\boldsymbol{F}$ is a continuous function $u:[0,+\infty) \rightarrow[0,+\infty)$ such that, for all $f \in \boldsymbol{F}$ and $t \in[0,+\infty)$,

$$
\|\mathcal{U}(t) f\| \leq u(t)\|f\| .
$$

Each strongly continuous linear semigroup admits an estimator of the form $u(t)=U e^{-B t}$, where $U \geq 1$ and $B$ are real constants: see [3].

Lipschitz maps. If $\mathcal{C}, \mathcal{D}$ are subsets of a topological vector space, we say that $\mathcal{C}$ is a strict subset of $\mathcal{D}$, and write $\mathcal{C} \Subset \mathcal{D}$, if $\mathcal{C}$ is bounded and $\overline{\mathcal{C}} \subset \mathcal{D}$, the symbol - denoting the closure. Now, let us be given a (possibly nonlinear) map, with open domain,

$$
\mathcal{P}: \operatorname{Dom} \mathcal{P} \subset \mathbf{F} \times \mathbf{R} \rightarrow \mathbf{F}, \quad(f, t) \mapsto \mathcal{P}(f, t) .
$$

2.2 Definition. We say that $\mathcal{P}$ is Lipschitz at fixed time (or, respectively, Lipschitz) on the strict subsets of its domain if, for every $\mathcal{C} \Subset D$ DomP , there is a nonnegative constant $L=L(\mathcal{C})$ (or, resp., a pair of nonnegative constants $L=L(\mathcal{C}), M=M(\mathcal{C})$ ) such that

$$
\begin{array}{r}
\left\|\mathcal{P}(f, t)-\mathcal{P}\left(f^{\prime}, t\right)\right\| \leq L\left\|f-f^{\prime}\right\| \quad \text { for }(f, t),\left(f^{\prime}, t\right) \in \mathcal{C} ; \\
\left\|\mathcal{P}(f, t)-\mathcal{P}\left(f^{\prime}, t^{\prime}\right)\right\| \leq L\left\|f-f^{\prime}\right\|+M\left|t-t^{\prime}\right| \quad \text { for }(f, t),\left(f^{\prime}, t^{\prime}\right) \in \mathcal{C} .
\end{array}
$$

Of course (2.12) implies (2.11) and the continuity of $\mathcal{P}$.

An example. Some applications presented in the sequel rely on a map $\mathcal{P}$ of the form

$$
\operatorname{Dom} \mathcal{P}=\mathbf{F} \times \Delta \quad(\Delta \subset \mathbf{R} \text { an open interval }), \quad \mathcal{P}(f, t):=\mathcal{P}(f, \ldots, f, t),
$$


where

$$
\mathcal{P}: \times{ }^{p} \mathbf{F} \times \Delta \rightarrow \mathbf{F} \quad(p \in\{1,2, \ldots\}), \quad\left(f_{1}, \ldots, f_{p}, t\right) \rightarrow \mathcal{P}\left(f_{1}, \ldots, f_{p}, t\right)
$$

is $\mathbf{R}$-linear in each argument $f_{1}, \ldots, f_{p}$; it is also assumed that

$$
\begin{gathered}
\left\|\mathcal{P}\left(f_{1}, \ldots, f_{p}, t\right)\right\| \leq P(t)\left\|f_{1}\right\| \ldots\left\|f_{p}\right\| \\
\left\|\mathcal{P}\left(f_{1}, \ldots, f_{p}, t\right)-\mathcal{P}\left(f_{1}, \ldots, f_{p}, t^{\prime}\right)\right\| \leq Q\left(t, t^{\prime}\right)\left\|f_{1}\right\| \ldots\left\|f_{p}\right\|
\end{gathered}
$$

for all $f_{1}, \ldots, f_{p} \in \mathbf{F}$ and $t, t^{\prime} \in \Delta$, where $P: \Delta \rightarrow[0,+\infty)$ and $Q: \Delta \times \Delta \rightarrow[0,+\infty)$ are continuous functions. It is finally required that, for each $B \Subset \Delta$, there is a constant $M=M(B)$ such that

$$
Q\left(t, t^{\prime}\right) \leq M\left|t-t^{\prime}\right| \quad \text { for } t, t^{\prime} \in B
$$

2.3 Proposition. With the previous assumptions, for all $f, f^{\prime} \in \boldsymbol{F}$ and $t, t^{\prime} \in \Delta$ it is

$$
\left\|\mathcal{P}(f, t)-\mathcal{P}\left(f^{\prime}, t^{\prime}\right)\right\| \leq P(t) \sum_{j=1}^{p}\left(\begin{array}{c}
p \\
j
\end{array}\right)\left\|f^{\prime}\right\|^{p-j}\left\|f-f^{\prime}\right\|^{j}+Q\left(t, t^{\prime}\right)\left\|f^{\prime}\right\|^{p} .
$$

Proof. Setting for convenience $f_{1}:=f^{\prime}, f_{2}:=f-f^{\prime}$ we can write

$$
\begin{gathered}
\mathcal{P}(f, t)-\mathcal{P}\left(f^{\prime}, t\right)=\mathcal{P}\left(f_{1}+f_{2}, \ldots, f_{1}+f_{2}, t\right)-\mathcal{P}\left(f_{1}, \ldots, f_{1}, t\right)= \\
=\sum_{j=1}^{p} \sum_{\left(l_{1}, \ldots, l_{p}\right) \in \Lambda_{p j}} \mathcal{P}\left(f_{l_{1}}, \ldots, f_{l_{p}}, t\right), \quad \Lambda_{p j}:=\left\{\left(l_{1}, \ldots, l_{p}\right) \in\{1,2\}^{p} \mid l_{s}=2 \text { for } j \text { values of } s\right\} .
\end{gathered}
$$

From the first inequality (2.15), we infer

$$
\left\|\mathcal{P}(f, t)-\mathcal{P}\left(f^{\prime}, t\right)\right\| \leq P(t) \sum_{j=1}^{p}\left(\begin{array}{c}
p \\
j
\end{array}\right)\left\|f^{\prime}\right\|^{p-j}\left\|f-f^{\prime}\right\|^{j},
$$

because $\Lambda_{p j}$ has cardinality $\left(\begin{array}{c}p \\ j\end{array}\right)$. Finally, the second assumption (2.15) gives

$$
\left\|\mathcal{P}\left(f^{\prime}, t\right)-\mathcal{P}\left(f^{\prime}, t^{\prime}\right)\right\| \leq Q\left(t, t^{\prime}\right)\left\|f^{\prime}\right\|^{p}
$$

and (2.19) (2.20), with the triangular inequality, yield the thesis (2.17).

Eq.(2.17) will be frequently used in the sequel; together with (2.16), it implies

2.4 Corollary. The map $\mathcal{P}$ is Lipschitz on the strict subsets of $\boldsymbol{F} \times \Delta$.

General formulation of the Volterra and Cauchy problems. We define formally both problems, and review their relations. 
2.5 Definition. Let us be given:

i) a strongly continuous linear semigroup $\mathcal{U}$ on $\boldsymbol{F}$;

ii) a continuous map $\mathcal{P}: \operatorname{Dom} \mathcal{P} \subset \boldsymbol{F} \times \boldsymbol{R} \rightarrow \boldsymbol{F}$, with open domain;

iii) a pair $\left(f_{0}, t_{0}\right) \in$ DomP .

The Volterra problem related to $\mathcal{U}$ and $\mathcal{P}$ with datum $f_{0}$ at time $t_{0}$ is the following one:

$$
\begin{gathered}
\text { Find } \varphi \in C\left(\left[t_{0}, t_{1} \mid, \boldsymbol{F}\right) \quad \text { such that graph } \varphi \subset \text { Dom } \mathcal{P}\right. \text { and } \\
\varphi(t)=\mathcal{U}\left(t-t_{0}\right) f_{0}+\int_{t_{0}}^{t} d s \mathcal{U}(t-s) \mathcal{P}(\varphi(s), s) \quad \text { for all } t \in\left[t_{0}, t_{1} \mid .\right.
\end{gathered}
$$

2.6 Definition. Consider:

i) a linear operator $\mathcal{A}: \operatorname{Dom} \mathcal{A} \subset \boldsymbol{F} \rightarrow \boldsymbol{F}$;

ii) a continuous map $\mathcal{P}$ as in the previous definition;

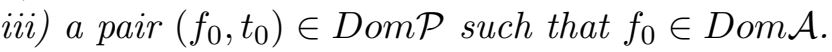

The Cauchy problem corresponding to $\mathcal{A}, \mathcal{P}$ with datum $f_{0}$ at time $t_{0}$ is the following one:

Find $\varphi \in C\left(\left[t_{0}, t_{1} \mid, D o m \mathcal{A}\right) \cap C^{1}\left(\left[t_{0}, t_{1} \mid, \boldsymbol{F}\right)\right.\right.$ such that graph $\varphi \subset$ DomP and

$$
\dot{\varphi}(t)=\mathcal{A} \varphi(t)+\mathcal{P}(\varphi(t), t) \quad \text { for all } t \in\left[t_{0}, t_{1} \mid, \quad \varphi\left(t_{0}\right)=f_{0} .\right.
$$

2.7 Proposition. Let $\mathcal{A}, \mathcal{P}, f_{0}, t_{0}$ be as in Def[2.6, and further assume $\mathcal{A}$ to be the generator of a strongly continuous linear semigroup $\mathcal{U}$. Then:

i) a solution $\varphi$ of the Cauchy problem (2.22) is also solution of the Volterra problem (2.21);

ii) as a partial converse, a solution $\varphi$ of the Volterra problem (2.21) is a solution of the Cauchy problem (2.22) in either of these situations: $\alpha) \boldsymbol{F}$ is reflexive and $\mathcal{P}$ is Lispchitz on the strict subsets of its domain; $\beta$ ) (trivial case) $\mathcal{A} \in \mathcal{L}(\boldsymbol{F})$, no further assumptions on $\boldsymbol{F}$ and $\mathcal{P}$.

Proof. It is essentially based on (2.7, 2.8): see 2] (conditions $\alpha) \beta$ ) in item ii) ensure a solution $\varphi \in C\left(\left[t_{0}, t_{1} \mid, \mathbf{F}\right)\right.$ of (2.21) to be in $C^{1}\left(\left[t_{0}, t_{1} \mid, \mathbf{F}\right) \cap C\left(\left[t_{0}, t_{1} \mid, D o m \mathcal{A}\right)\right)\right.$.

In particular, the operator $\mathcal{A}:=0$ is the generator of the identity semigroup $\mathcal{U}(t)=\mathbf{1}_{\mathbf{F}}$ for all $t$. With this remark, the framework of this paper applies to any ODE $\dot{\varphi}(t)=\mathcal{P}(\varphi(t), t)$ in a Banach space, also including the finite dimensional cases $\mathbf{F}=\mathbf{R}^{m}$ or $\mathbf{C}^{m}$.

2.8 Proposition. Consider the Volterra problem (2.21), where $\mathcal{U}$ is a strongly continuous linear semigroup, and $\mathcal{P}$ is continuous and Lipschitz at fixed time on the strict subsets of its domain; then i) ii) hold.

i) Problem (2.21) has a solution.

ii) If $\varphi:\left[t_{0}, t_{1} \mid \rightarrow \boldsymbol{F}\right.$ and $\varphi^{\prime}:\left[t_{0}, t_{1}^{\prime} \mid \rightarrow \boldsymbol{F}\right.$ are two solutions, it is

$$
\varphi(t)=\varphi^{\prime}(t) \quad \text { for } t \in\left[t_{0}, t_{1} \mid \cap\left[t_{0}, t_{1}^{\prime} \mid .\right.\right.
$$


Proof. ii) We consider any $t_{2}$ in the intersection of the domains. Subtracting Eq.(2.21) for $\varphi$ from the analogous equation for $\varphi^{\prime}$, and taking the norm, we obtain

$$
\left\|\varphi(t)-\varphi^{\prime}(t)\right\| \leq \int_{t_{0}}^{t} d s u(t-s)\left\|\mathcal{P}(\varphi(s), s)-\mathcal{P}\left(\varphi^{\prime}(s), s\right)\right\| \leq U L \int_{t_{0}}^{t}\left\|\varphi(s)-\varphi^{\prime}(s)\right\|
$$

for each $t \in\left[t_{0}, t_{2}\right]$. Here: $u$ is any estimator for $\mathcal{U} ; U:=\max _{s \in\left[t_{0}, t_{2}\right]} u(s) ; L$ is a constant fulfilling the Lipschitz condition (2.11) for $\mathcal{P}$ on the set $\mathcal{C}:=\operatorname{graph}\left(\varphi^{\prime} \uparrow\left[t_{0}, t_{2}\right]\right) \cup \operatorname{graph}(\varphi \uparrow$ $\left.\left[t_{0}, t_{2}\right]\right)$ (this $\mathcal{C}$ is a strict subset of $\operatorname{Dom} \mathcal{P}$ ). Eq.(2.24) and the classical Gronwall Lemma [10] imply $\left\|\varphi(t)-\varphi^{\prime}(t)\right\|=0$ for all $t \in\left[t_{0}, t_{2}\right]$.

i) Eq.(2.21) is the fixed point problem for a Volterra type integral operator, and a solution can be constructed by standard Peano-Picard iteration, starting from the function $\varphi_{0}(t):=$ const. : $=f_{0}$; see, e.g., [2].

From our viewpoint, the previously mentioned argument for local existence is a particular case of a more general statement, allowing to construct a solution of (2.21) by a PeanoPicard iteration with starting point any approximate solution (of sufficiently small error); all this will be discussed in the next Section.

Of course, Prop 2.7 allows to transfer the statements on uniqueness and existence from (2.21) to (2.22). Let us call maximal a solution $\varphi$ of (2.21) or (2.22) which has no proper extension. If one can grant the existence of a solution on a sufficiently small interval, and the coincidence of two solutions on the intersection of their domains, it follows that a unique maximal solution $\varphi$ exists and any other solution is a proper restriction of the maximal one. Furthermore, if local existence is granted for arbitray data, the domain of the maximal solution $\varphi$ with a given datum has the form $\left[t_{0}, \vartheta\right.$ ) (otherwise, $\varphi$ could be extended taking its value at $\vartheta$ as a new initial datum).

\section{Approximate solutions. Statements of the main results.}

We consider a strongly continuous linear semigroup $\mathcal{U}$ on the Banach space $\mathbf{F}$, and a continuous function $\mathcal{P}: \operatorname{Dom} \mathcal{P} \subset \mathbf{F} \times \mathbf{R} \rightarrow \mathbf{F}$ with open domain. We are interested in the Volterra problem (2.21), for a given pair $\left(f_{0}, t_{0}\right) \in \operatorname{Dom} \mathcal{P}$.

3.1 Definition. By an approximate solution of problem (2.21), we mean any continuous function $\varphi_{a p}:\left[t_{0}, t_{1} \mid \rightarrow \boldsymbol{F}\right.$, such that graph $\varphi_{a p} \subset$ DomP $\mathcal{P}$. Given any such function, we stipulate the following:

i) the integral error of $\varphi_{a p}$ is the function

$$
E\left(\varphi_{a p}\right): t \in\left[t_{0}, t_{1} \mid \mapsto E\left(\varphi_{a p}\right)(t):=\varphi_{a p}(t)-\mathcal{U}\left(t-t_{0}\right) f_{0}-\int_{t_{0}}^{t} d s \mathcal{U}(t-s) \mathcal{P}\left(\varphi_{a p}(s), s\right) ;\right.
$$

an integral error estimator for $\varphi_{a p}$ is a continuous function $\mathcal{E}:\left[t_{0}, t_{1} \mid \rightarrow[0,+\infty)\right.$ such that, for all $t$ in this interval,

$$
\left\|E\left(\varphi_{a p}\right)(t)\right\| \leq \mathcal{E}(t)
$$


ii) The datum error for $\varphi_{a p}$ is the difference

$$
d\left(\varphi_{a p}\right):=\varphi_{a p}\left(t_{0}\right)-f_{0}
$$

a datum error estimator for $\varphi_{\text {ap }}$ is a nonnegative real number $\delta$ such that

$$
\left\|d\left(\varphi_{a p}\right)\right\| \leq \delta .
$$

iii) If $\mathcal{A}$ is the generator of $\mathcal{U}$ and $\varphi_{a p} \in C\left(\left[t_{0}, t_{1} \mid, D o m \mathcal{A}\right) \cap C^{1}\left(\left[t_{0}, t_{1} \mid, \boldsymbol{F}\right)\right.\right.$, the differential error of $\varphi_{a p}$ is the function

$$
e\left(\varphi_{a p}\right): t \in\left[t_{0}, t_{1} \mid \mapsto e\left(\varphi_{a p}\right)(t):=\dot{\varphi}_{a p}(t)-\mathcal{A} \varphi_{a p}(t)-\mathcal{P}\left(\varphi_{a p}(t), t\right) ;\right.
$$

a differential error estimator for $\varphi_{a p}$ is a continuous function $\epsilon:\left[t_{0}, t_{1} \mid \rightarrow[0,+\infty)\right.$ such that, for $t$ in this interval,

$$
\left\|e\left(\varphi_{a p}\right)(t)\right\| \leq \epsilon(t) .
$$

Of course, $\varphi_{a p}$ is a solution of the Volterra (resp., Cauchy) problem iff $E\left(\varphi_{a p}\right)=0$ (resp., $d\left(\varphi_{a p}\right)=0$ and $\left.e\left(\varphi_{a p}\right)=0\right)$.

3.2 Lemma. Let $\varphi_{a p}:\left[t_{0}, t_{1} \mid \rightarrow \boldsymbol{F}\right.$ be an approximate solution of the Volterra problem (2.21), and assume the regularity conditions in item iii) of the previous Definition. Then, the integral error of $\varphi_{a p}$ is related to the datum and differential errors by

$$
E\left(\varphi_{a p}\right)(t)=\mathcal{U}\left(t-t_{0}\right) d\left(\varphi_{a p}\right)+\int_{t_{0}}^{t} d s \mathcal{U}(t-s) e\left(\varphi_{a p}\right)(s)
$$

If $\mathcal{U}, d\left(\varphi_{a p}\right), e\left(\varphi_{a p}\right)$ have estimators $u, \delta, \epsilon$, then $E\left(\varphi_{a p}\right)$ has the estimator

$$
\mathcal{E}(t):=u\left(t-t_{0}\right) \delta+\int_{t_{0}}^{t} d s u(t-s) \epsilon(s) \quad \text { for all } t \in\left[t_{0}, t_{1} \mid .\right.
$$

Proof. Eq. (3.7) follows applying the definitions of $E\left(\varphi_{a p}\right), d\left(\varphi_{a p}\right), e\left(\varphi_{a p}\right)$ and the identity (2.8) with $\psi:=\varphi_{a p}$. Given (3.7), the bound (3.8) on $\left\|E\left(\varphi_{a p}\right)(t)\right\|$ is evident.

Remark. The estimator $\mathcal{E}$ defined by (3.8) is useful, because in many cases it can be easily computed. However, in peculiar situations involving oscillating functions, this estimator can be rough. For example, consider the semigroup $\mathcal{U}(t):=\mathbf{1}_{\mathbf{F}}$ for all $t$, with generator $\mathcal{A}=0$ and estimator $u(t):=1$. Let us choose $\varphi_{a p}(t):=f_{0}$ for all $t$, so that $d\left(\varphi_{a p}\right)=0$, $e\left(\varphi_{a p}\right)(t)=-\mathcal{P}\left(f_{0}, t\right)$ and $E\left(\varphi_{a p}\right)(t)=-\int_{t_{0}}^{t} d s \mathcal{P}\left(f_{0}, s\right)$. Suppose $\mathcal{P}\left(f_{0}, t\right)=g_{0} e^{i \omega t}$, with $\omega \in(0,+\infty)$ and $g_{0}$ a vector of the (complex) space $\mathbf{F}$; then, the best estimators for $d\left(\varphi_{a p}\right)$ and $e\left(\varphi_{a p}\right)$ are, respectively, $\delta=0$ and $\epsilon(t)=\left\|g_{0}\right\|$. Correspondingly, Eq.(3.8) gives the integral error estimator $\mathcal{E}(t)=\left\|g_{0}\right\|\left(t-t_{0}\right)$; on the other hand, it is found by direct computation that $E\left(\varphi_{a p}\right)(t)=i g_{0}\left(e^{i \omega t}-e^{i \omega t_{0}}\right) / \omega$; thus $\left\|E\left(\varphi_{a p}\right)(t)\right\|$ is a bounded function of $t$, whereas the estimator $\mathcal{E}$ grows linearly.

Similar drawbacks of the estimator (3.8) in the presence of oscillatory functions are met (even for $\mathbf{F}=\mathbf{R}^{m}$ ) if one considers a differential equation with fast periodic variables and the approximate solutions which arise from averaging methods [8]. 
To formulate the main theorem on approximate solutions, we need one more notion describing the growth of $\mathcal{P}$ away from a function $\psi:\left[t_{0}, t_{1} \mid \rightarrow \mathbf{F}\right.$, such that graph $\psi \subset$ Dom $\mathcal{P}$.

3.3 Definition. A growth estimator for $\mathcal{P}$ from $\psi$ (if it exists) is a continuous function

$$
\ell:[0, \rho) \times\left[t_{0}, t_{1} \mid \rightarrow[0,+\infty), \quad(r, t) \mapsto \ell(r, t)\right.
$$

such that:

i) $\rho \in(0,+\infty]$ and $\boldsymbol{T}(\psi, \rho) \subset \operatorname{Dom} \mathcal{P}$ (see Eq.(2.3));

ii) $\ell$ is nondecreasing in the first variable: $\ell(r, t) \leq \ell\left(r^{\prime}, t\right)$ for $r \leq r^{\prime}$;

iii) for all $(f, t) \in \boldsymbol{T}(\psi, \rho)$, it is

$$
\|\mathcal{P}(f, t)-\mathcal{P}(\psi(t), t)\| \leq \ell(\|f-\psi(t)\|, t) .
$$

Remarks. a) From a continuous function $\ell^{\prime}:[0, \rho) \times\left[t_{0}, t_{1} \mid \rightarrow[0,+\infty)\right.$ fulfilling i) iii) but not ii), we can construct the function $\ell(r, t):=\max _{r^{\prime} \in[0, r]} \ell^{\prime}\left(r^{\prime}, t\right)$, which also fulfils ii).

b) A function $\mathcal{P}$ which is Lipschitz at fixed time on a tube around $\psi$ possesses on it a growth estimator linear in $r$. Less trivial estimators appear if $\operatorname{Dom} \mathcal{P}=\mathbf{F} \times \Delta, \Delta$ a real interval, and one wishes to estimate the growth of $\mathcal{P}$ from $\psi$ on the whole product space $\mathbf{F} \times\left[t_{0}, t_{1} \mid\right.$ (= on a tube of infinite radius). For instance, consider a map $\mathcal{P}$ as in the Example of page 4 the growth of $\mathcal{P}$ from any $\psi:\left[t_{0}, t_{1} \mid \subset \Delta \rightarrow \mathbf{F}\right.$ admits the estimator

$$
\ell(r, t):=P(t) \sum_{j=1}^{p}\left(\begin{array}{l}
p \\
j
\end{array}\right)\|\psi(t)\|^{p-j} r^{j}
$$

$\left(r \in[0,+\infty), t \in\left[t_{0}, t_{1} \mid\right)\right.$. To find this, apply Eq.(2.17) with $f^{\prime}=\psi(t)$ and $t^{\prime}=t$.

We come to the main theorem of this Section: the proof will be given in Sect 4

3.4 Proposition. Let us be given a Volterra problem (2.21), where: $\mathcal{U}$ is a strongly continuous linear semigroup; $\mathcal{P}:$ DomP $\subset \boldsymbol{F} \times \boldsymbol{R} \rightarrow \boldsymbol{R}$ is continuous and Lipschitz at fixed time on the strict subsets of its open domain (Def. 2.2). Assume that:

i) $u$ is an estimator for $\mathcal{U}$;

ii) $\varphi_{a p}:\left[t_{0}, t_{1} \mid \rightarrow \boldsymbol{F}\right.$ is an approximate solution of (2.21), and $\mathcal{E}:\left[t_{0}, t_{1} \mid \rightarrow[0,+\infty)\right.$ is an estimator for the integral error $E\left(\varphi_{a p}\right)$;

iii) $\ell:[0, \rho) \times\left[t_{0}, t_{1} \mid \rightarrow[0,+\infty)\right.$ is a growth estimator for $\mathcal{P}$ from $\varphi_{a p}(\rho \in(0,+\infty])$.

Consider the following problem:

$$
\begin{gathered}
\text { Find } R \in C\left(\left[t_{0}, t_{1} \mid,[0, \rho)\right) \quad\right. \text { such that } \\
\mathcal{E}(t)+\int_{t_{0}}^{t} d s u(t-s) \ell(R(s), s) \leq R(t) \quad \text { for } t \in\left[t_{0}, t_{1} \mid .\right.
\end{gathered}
$$

If (3.12) has a solution $R$ with domain $\left[t_{0}, t_{1} \mid\right.$, then (2.21) has a solution $\varphi$ with the same domain, and for all $t$ therein it is

$$
\left\|\varphi(t)-\varphi_{a p}(t)\right\| \leq R(t) .
$$

The solution $\varphi$ can be constructed by a Peano-Picard iteration, starting from $\varphi_{a p}$. 
3.5 Definition. Eq. (3.12) will be referred to as the control inequality.

Remarks. i) The function $R$ is required to exist on the same domain $\left[t_{0}, t_{1} \mid\right.$ of $\varphi_{a p}$. In many applications, one starts with an approximate solution on a domain $\left[t_{0}, t_{2} \mid\right.$ and then finds (3.12) to a have a solution $R$ on a domain $\left[t_{0}, t_{1} \mid \subset\left[t_{0}, t_{2} \mid\right.\right.$; of course, in this case the previous Proposition must be applied to $\varphi_{a p}\left\lceil\left[t_{0}, t_{1} \mid\right.\right.$.

ii) As anticipated, the argument we will employ to prove Prop 3.4 is different from the "continuation principle" mentioned in the Introduction. Instead of using a Gronwall Lemma plus a reductio ad absurdum, we will prove the existence of $\varphi$ on $\left[t_{0}, t_{1} \mid\right.$, and the bound (3.12), by a constructive Peano-Picard iteration; the convergence of this iteration on the whole $\left[t_{0}, t_{1} \mid\right.$ has some theoretical interest by itself. Furthermore, this approach overcomes some technicalities required by the application of nonlinear Gronwall lemmas (the analysis of the associated integral equation, and the necessity to determine the greatest solution when uniqueness fails [16]).

Apart from the general concept of approximate solution employed here, the idea to prove existence for an ODE $\dot{f}=\mathcal{P}(f, t)$ by the Peano-Picard method, under conditions of nonlinear growth for $\mathcal{P}$ of more global type than the Lipschitz property can be ascribed to Caratheodory [1, and was developed in 17] 12.

iii) Of course, we can accept as a solution of (3.12) an $R$ fulfilling the equation

$$
\mathcal{E}(t)+\int_{t_{0}}^{t} d s u(t-s) \ell(R(s), s)=R(t) \quad \text { for } t \in\left[t_{0}, t_{1} \mid,\right.
$$

hereafter referred to as the control integral equation. (The existence of such an $R$ on a sufficiently short interval is granted by standard compactness arguments, see [16. Uniqueness can be proved under supplementary assumptions of Lipschitz kind for $\ell$ ).

Let us exploit a typical case, where the control integral equation (3.14) is equivalent to a Cauchy problem. To this purpose, assume that

$$
u(t)=U e^{-B t} \quad(U \geq 1, B \in \mathbf{R}), \quad \mathcal{E}(t)=U e^{-B\left(t-t_{0}\right)} \delta+U \int_{t_{0}}^{t} d s e^{-B(t-s)} \epsilon(s)
$$

for some constant $\delta \geq 0$ and some continuous function $\epsilon:\left[t_{0}, t_{1} \mid \rightarrow[0,+\infty)\right.$ (for example, the estimator $\mathcal{E}$ derived from Eq.(3.8) has the above form). Then, multiplying by $e^{B\left(t-t_{0}\right)}$ we see that Eq.(3.14) is equivalent to

$$
U \delta+U \int_{t_{0}}^{t} d s e^{B\left(s-t_{0}\right)} \epsilon(s)+U \int_{t_{0}}^{t} d s e^{B\left(s-t_{0}\right)} \ell(R(s), s)=e^{B\left(t-t_{0}\right)} R(t) .
$$

Any solution $R$ of (3.16) is clearly $C^{1}$. By derivation in $t$ of this equation, and evaluation of the same at $t=t_{0}$, we get

3.6 Proposition. If $u$ and $\mathcal{E}$ are as in (3.15), Eq. (3.14) is equivalent to the problem

$$
\dot{R}(t)=U \epsilon(t)+U \ell(R(t), t)-B R(t), \quad R\left(t_{0}\right)=U \delta,
$$

for an unknown function $R \in C^{1}\left(\left[t_{0}, t_{1} \mid,[0, \rho)\right)\right.$ (the terms control problem, or control equation will be employed as well, for (3.17) or the differential equation therein). 


\section{Proof of Prop.3.4.}

We present in detail the argument in the case of a compact interval $\left[t_{0}, t_{1}\right]$. In this case, we use the space $C\left(\left[t_{0}, t_{1}\right], \mathbf{F}\right)$, regarded as a Banach space with the usual sup norm $\|\psi\|:=\max _{t \in\left[t_{0}, t_{1}\right]}\|\psi(t)\|$. The case when $\varphi_{a p}, R$, etc. are defined on $\left[t_{0}, t_{1}\right.$ ) (with $t_{1}$ possibly infinite) is treated in a similar way, using $C\left(\left[t_{0}, t_{1}\right), \mathbf{F}\right)$ with the topology of uniform convergence on all compact subintervals $\left[t_{0}, \tau\right] \subset\left[t_{0}, t_{1}\right)\left({ }^{1}\right)$. Sticking from now on to the case $\left[t_{0}, t_{1}\right]$, we introduce the objects

$$
\begin{gathered}
\varrho:=\max _{t \in\left[t_{0}, t_{1}\right]} R(t) ; \quad \overline{\mathbf{T}}\left(\varphi_{a p}, \varrho\right)=\left\{(f, t) \in \mathbf{F} \times\left[t_{0}, t_{1}\right] \mid\left\|f-\varphi_{a p}(t)\right\| \leq \varrho\right\} ; \\
\mathfrak{D}:=\left\{\psi \in C\left(\left[t_{0}, t_{1}\right], \mathbf{F}\right) \mid\left\|\psi(t)-\varphi_{a p}(t)\right\| \leq R(t) \text { for } t \in\left[t_{0}, t_{1}\right]\right\} ;
\end{gathered}
$$

then $\varrho<\rho$, and $\overline{\mathbf{T}}\left(\varphi_{a p}, \varrho\right) \subset \mathbf{T}\left(\varphi_{a p}, \rho\right)$ is a strict subset of DomP. $\mathfrak{D}$ is a closed subset of $C\left(\left[t_{0}, t_{1}\right], \mathbf{F}\right)$ (containing $\left.\varphi_{a p}\right)$ and $\psi \in \mathfrak{D} \Rightarrow \operatorname{graph} \psi \subset \overline{\mathbf{T}}\left(\varphi_{a p}, \varrho\right)$.

\subsection{Definition. We put}

$$
\begin{gathered}
\mathcal{J}: \mathfrak{D} \rightarrow C\left(\left[t_{0}, t_{1}\right], \boldsymbol{F}\right), \quad \psi \mapsto \mathcal{J}(\psi), \\
\mathcal{J}(\psi)(t):=\mathcal{U}\left(t-t_{0}\right) f_{0}+\int_{t_{0}}^{t} d s \mathcal{U}(t-s) \mathcal{P}(\psi(s), s) \quad \forall t \in\left[t_{0}, t_{1}\right] .
\end{gathered}
$$

Of course, we have

4.2 Lemma. $\varphi \in \mathfrak{D}$ solves the Volterra problem (2.21) if and only if $\varphi=\mathcal{J}(\varphi)$.

4.3 Lemma. There is a constant $\Lambda \geq 0$ such that, for all $\psi, \psi^{\prime} \in \mathfrak{D}$,

$$
\left\|\mathcal{J}(\psi)(t)-\mathcal{J}\left(\psi^{\prime}\right)(t)\right\| \leq \Lambda \int_{t_{0}}^{t} d s\left\|\psi(s)-\psi^{\prime}(s)\right\| \quad \text { for } t \in\left[t_{0}, t_{1}\right] .
$$

Thus $\left\|\mathcal{J}(\psi)-\mathcal{J}\left(\psi^{\prime}\right)\right\| \leq \Lambda\left(t_{1}-t_{0}\right)\left\|\psi-\psi^{\prime}\right\|$, which implies the continuity of $\mathcal{J}$.

Proof. $\mathcal{P}$ is Lipschitz at fixed time on the strict subsets of its domain, so there is a constant $L \geq 0$ such that $\left\|\mathcal{P}(f, t)-\mathcal{P}\left(f^{\prime}, t\right)\right\| \leq L\left\|f-f^{\prime}\right\|$ for $(f, t),\left(f^{\prime}, t\right) \in \overline{\mathbf{T}}\left(\varphi_{a p}, \varrho\right)$. If $u$ is an estimator for the semigroup $\mathcal{U}$ and $U:=\max _{t \in\left[t_{0}, t_{1}\right]} u(t)$, we see that Eq.(4.4) is fulfilled with $\Lambda:=U L$; the remaining statements are trivial.

\subsection{Lemma. $\mathcal{J}(\mathfrak{D}) \subset \mathfrak{D}$.}

\footnotetext{
${ }^{1}$ This complete, locally convex topology on $C\left(\left[t_{0}, t_{1}\right), \mathbf{F}\right)$ is defined by the seminorms $\left(\|\|_{\tau}\right)_{\tau \in\left[t_{0}, t_{1}\right)}$ where $\|\psi\|_{\tau}:=\max _{t \in\left[t_{0}, \tau\right]}\|\psi(t)\|$. To adapt the proof to this case, the objects $\varrho, \overline{\mathbf{T}}\left(\varphi_{a p}, \varrho\right), \Lambda$, etc. appearing in the sequel must be replaced by families of objects $\varrho_{\tau}, \overline{\mathbf{T}}\left(\varphi_{a p} \uparrow\left[t_{0}, \tau\right], \varrho_{\tau}\right), \Lambda_{\tau}$, etc., one for each $\tau$; the definition of $\mathfrak{D}$ is simply rephrased using $\left[t_{0}, t_{1}\right)$. The Peano-Picard iteration converges in all the seminorms \|\|$_{\tau}$.
} 
Proof. Let $\psi \in \mathfrak{D}$. For all $t \in\left[t_{0}, t_{1}\right]$ the definitions of $\mathcal{J}$ and of the error $E\left(\varphi_{a p}\right)$, with the properties of $\mathcal{E}, u, \ell$, imply

$$
\begin{gathered}
\mathcal{J}(\psi)(t)-\varphi_{a p}(t)=-E\left(\varphi_{a p}\right)(t)+\int_{t_{0}}^{t} d s \mathcal{U}(t-s)\left[\mathcal{P}(\psi(s), s)-\mathcal{P}\left(\varphi_{a p}(s), s\right)\right], \\
\left\|\mathcal{J}(\psi)(t)-\varphi_{a p}(t)\right\| \leq \mathcal{E}(t)+\int_{t_{0}}^{t} d s u(t-s) \ell\left(\left\|\psi(s)-\varphi_{a p}(s)\right\|, s\right) .
\end{gathered}
$$

On the other hand, $\left\|\psi(s)-\varphi_{a p}(s)\right\| \leq R(s)$ which implies $\ell\left(\left\|\psi(s)-\varphi_{a p}(s)\right\|, s\right) \leq \ell(R(s), s)$; inserting this into (4.6), and using the control inequality (3.12) for $R$, we conclude

$$
\left\|\mathcal{J}(\psi)(t)-\varphi_{a p}(t)\right\| \leq R(t), \quad \text { i.e., } \quad \mathcal{J}(\psi) \in \mathfrak{D} .
$$

The invariance of $\mathfrak{D}$ under $\mathcal{J}$ is a central result; with the previously shown properties of $\mathcal{J}$, it allows to set up the Peano-Picard iteration and get ultimately a fixed point.

4.5 Definition. $\left(\varphi_{k}\right)(k \in \boldsymbol{N})$ is the sequence of functions in $\mathfrak{D}$, defined recursively by

$$
\varphi_{0}:=\varphi_{a p}, \quad \varphi_{k}:=\mathcal{J}\left(\varphi_{k-1}\right) \quad(k \geq 1) .
$$

4.6 Lemma. For all $k \in N$ and $t \in\left[t_{0}, t_{1}\right]$, it is

$$
\left\|\varphi_{k+1}(t)-\varphi_{k}(t)\right\| \leq \Sigma \frac{\Lambda^{k}\left(t-t_{0}\right)^{k}}{k !}
$$

where $\Lambda$ is the constant of $E q$. 4.4] and $\Sigma:=\max _{t \in\left[t_{0}, t_{1}\right]} \mathcal{E}(t)$. So,

$$
\left\|\varphi_{k+1}-\varphi_{k}\right\| \leq \Sigma \frac{\Lambda^{k}\left(t_{1}-t_{0}\right)^{k}}{k !}
$$

Proof. Eq. (4.10) is an obvious consequence of (4.9). We will prove (4.9) by recursion, indicating with a subscript ${ }_{k}$ the thesis at a specified order.

We have $\varphi_{1}(t)-\varphi_{0}(t)=\mathcal{J}\left(\varphi_{a p}\right)(t)-\varphi_{a p}(t)=-E\left(\varphi_{a p}\right)(t)$ by the definition of $E\left(\varphi_{a p}\right)$, whence $\left\|\varphi_{1}(t)-\varphi_{0}(t)\right\| \leq \mathcal{E}(t) \leq \Sigma$; this gives (4.9) 0 . For each $k \geq 0$, we have

$$
\left\|\varphi_{k+2}(t)-\varphi_{k+1}(t)\right\|=\left\|\mathcal{J}\left(\varphi_{k+1}\right)(t)-\mathcal{J}\left(\varphi_{k}\right)(t)\right\| \leq \Lambda \int_{t_{0}}^{t} d s\left\|\varphi_{k+1}(s)-\varphi_{k}(s)\right\|
$$

the last passage depending on Eq.(4.4). Eq.s (4.11) and (4.9) $k$ imply (4.9) $k+1$.

4.7 Lemma. For all $k, k^{\prime}$ and $n \in \boldsymbol{N}$, it is

$$
\left\|\varphi_{k^{\prime}}-\varphi_{k}\right\| \leq \Sigma e^{\Lambda\left(t_{1}-t_{0}\right)} \frac{\Lambda^{h}\left(t_{1}-t_{0}\right)^{h}}{h !}, \quad h:=\min \left(k, k^{\prime}\right)
$$

so, $\left(\varphi_{k}\right)$ is a Cauchy sequence. 
Proof. To prove Eq.(4.12), it suffices to consider the case $k^{\prime}>k$ (so that $h=k$ ). Writing $\varphi_{k^{\prime}}-\varphi_{k}=\sum_{j=k}^{k^{\prime}-1}\left(\varphi_{j+1}-\varphi_{j}\right)$ and using Eq.(4.10) we get

$$
\left\|\varphi_{k^{\prime}}-\varphi_{k}\right\| \leq \Sigma \sum_{j=k}^{k^{\prime}-1} \frac{\Lambda^{j}\left(t_{1}-t_{0}\right)^{j}}{j !} .
$$

On the other hand, for each $\xi \geq 0$, it is $\sum_{j=k}^{k^{\prime}-1} \xi^{j} / j ! \leq \sum_{j=k}^{+\infty} \xi^{j} / j ! \leq e^{\xi} \xi^{k} / k !$; with $\xi=\Lambda\left(t_{1}-t_{0}\right)$ we obtain Eq.4.12), implying $\left\|\varphi_{k^{\prime}}-\varphi_{k}\right\| \rightarrow 0$ for $\left(k, k^{\prime}\right) \rightarrow \infty\left({ }^{2}\right)$. $\diamond$

Proof of Prop 3.4. $\left(\varphi_{k}\right)$ being a Cauchy sequence, $\lim _{k \mapsto+\infty} \varphi_{k}:=\varphi$ exists in $C\left(\left[t_{0}, t_{1}\right], \mathbf{F}\right)$; $\varphi$ belongs to $\mathfrak{D}$ because this set is closed. By the continuity of $\mathcal{J}$, we have

$$
\mathcal{J}(\varphi)=\lim _{k \mapsto+\infty} \mathcal{J}\left(\varphi_{k}\right)=\lim _{k \mapsto+\infty} \varphi_{k+1}=\varphi ;
$$

thus, $\varphi$ solves the Volterra problem (2.21). Finally, the inequality $\left\|\varphi(t)-\varphi_{a p}(t)\right\| \leq R(t)$ for all $t \in\left[t_{0}, t_{1}\right]$ is ensured by the definition of $\mathfrak{D}$.

\section{An elementary application of Prop.3.4.}

The results we are presenting in the forthcoming Prop.s [5.1 5.2 are essentially known (see, e.g., [7] for the case $\mathcal{U}(t)=\mathbf{1}$ and $\operatorname{dim} \mathbf{F}$ finite), but their derivation as a subcase of Prop 3.4 is instructive: the main idea is to use the zero function as an approximate solution.

5.1 Proposition. Consider the Volterra problem (2.21), where:

i) $\mathcal{U}$ is a strongly continuous linear semigroup, with an estimator $u(t):=U e^{-B t}(U \geq 1$, $B \geq 0$ ).

ii) $\mathcal{P}$ is continuous and Lipschitz at fixed time on the strict subsets of its open domain. It is $\boldsymbol{B}(0, \rho) \times\left[t_{0}, T\right) \subset$ DomP for some $\rho \in(0,+\infty], T \in\left(t_{0},+\infty\right]$, and

$$
\mathcal{P}(0, t)=0 \quad \text { for } t \in\left[t_{0}, T\right) .
$$

There is a continuous function $\ell:[0, \rho) \times\left[t_{0}, T\right) \rightarrow[0,+\infty)$, non decreasing in the first variable, such that

$$
\|\mathcal{P}(f, t)\| \leq \ell(\|f\|, t) \quad \text { for }(f, t) \in \boldsymbol{B}(0, \rho) \times\left[t_{0}, T\right) .
$$

iii) The control problem

$$
\dot{R}(t)=U \ell(R(t), t)-B R(t), \quad R\left(t_{0}\right)=U\left\|f_{0}\right\|,
$$

has a solution $R \in C^{1}\left(\left[t_{0}, t_{N}\right),[0, \rho)\right)$, for some $t_{N} \in\left(t_{0}, T\right)$.

Then, the Volterra problem (2.21) has a solution $\varphi \in C\left(\left[t_{0}, t_{N}\right), \boldsymbol{F}\right)$ and, for all $t$ in this interval,

$$
\|\varphi(t)\| \leq R(t) .
$$

\footnotetext{
${ }^{2}$ Incidentally we note that (4.12) could be improved, but this is unnecessary: this estimate is needed only to infer the Cauchy property of the sequence.
} 
Proof. We apply Prop.s [3.4 [3.6] with $\varphi_{a p}(t):=0$ for $t \in\left[t_{0}, t_{N}\right)$; the function $\ell$ in item ii) is a growth estimator for $\mathcal{P}$ from the approximate solution. The datum and differential errors are $d\left(\varphi_{a p}\right)=-f_{0}, e\left(\varphi_{a p}\right)(t)=0$, so they admit the estimators $\delta:=\left\|f_{0}\right\|, \epsilon(t):=0$. With these estimators, problem (3.17) takes the form (5.3).

The symbol $t_{N}$ adopted here for the right extreme of the domain of $R$ is chosen for future convenience; it emphasizes the dependence of this object on the norm of the initial datum. In the time independent case $\ell(r, t)=\ell(r)$, Eq. (5.3) can be solved by the quadrature formula

$$
\int_{U\left\|f_{0}\right\|}^{R(t)} \frac{d r}{U \ell(r)-B r}=t-t_{0}
$$

let us write the explicit solution in a simple case.

5.2 Proposition. Let the previous assumptions be satisfied with $t_{0}=0, \delta=+\infty$, $T=+\infty$ and $\ell(r, t)=\operatorname{Pr}^{p}(P \geq 0, p>1)$. Then, the problem (5.3) has the solution $R \in C^{1}\left(\left[0, t_{N}\right),[0,+\infty)\right)$ defined hereafter. It is

$$
\begin{gathered}
t_{N}:= \begin{cases}+\infty & \text { if } P U^{p}\left\|f_{0}\right\|^{p-1} \leq B, \\
\frac{1}{(p-1)} L_{B}\left(P U^{p}\left\|f_{0}\right\|^{p-1}\right) & \text { if } P U^{p}\left\|f_{0}\right\|^{p-1}>B,\end{cases} \\
L_{B}(u):= \begin{cases}-(1 / B) \log (1-B / u) & \text { if } 0<B<u, \\
1 / u & \text { if } B=0<u ;\end{cases} \\
R(t):=\frac{\text { for all } t \in\left[0, t_{N}\right),}{\left[1-\left(P U^{p}\left\|f_{0}\right\|^{p-1}-B\right) E_{B}((p-1) t)\right]^{\frac{1}{p-1}}} \quad \text { if } B>0, \\
E_{B}(u):= \begin{cases}\frac{e^{B u}-1}{B} & \text { if } B=0 . \\
u & P \text { if }\end{cases}
\end{gathered}
$$

The function $R$ has the following features. If $P U^{p}\left\|f_{0}\right\|^{p-1}<B, R$ is decreasing and $R(t) \rightarrow 0$ for $t \rightarrow+\infty$. If $P U^{p}\left\|f_{0}\right\|^{p-1}=B, R(t)=$ const. $=U\left\|f_{0}\right\|$. If $P U^{p}\left\|f_{0}\right\|^{p-1}>B$, $R$ is increasing and $R(t) \rightarrow+\infty$ for $t \rightarrow t_{N}^{-}$.

Proof. Everything follows in an elementary way from (5.5).

Remarks. i) A map $\mathcal{P}$ as in the Example of page 4 has the properties required by the previous Proposition, if the function $t \mapsto P(t)$ appearing in Eq.(2.15) is bounded on the interval $\left[t_{0}, T\right)$ under consideration. In this case, the growth of $\mathcal{P}$ from zero admits the estimator $\ell(r, t):=\operatorname{Pr}^{p}$, with $P$ the sup of the function $t \mapsto P(t)$.

ii) Obviously enough: if $P U^{p}\left\|f_{0}\right\|^{p-1}<B$, the Volterra problem (2.21) has a solution $\varphi$ defined for all $t \in[0,+\infty)$, and $\|\varphi(t)\| \leq R(t) \rightarrow 0$ for $t \rightarrow+\infty$. If $P U^{p}\left\|f_{0}\right\|^{p-1}=B$, we have again a solution defined on $[0,+\infty)$, and $\|\varphi(t)\| \leq U\left\|f_{0}\right\|$ for all $t$. If $P U^{p}\left\|f_{0}\right\|^{p-1}>$ $B$, we can grant existence of a solution $\varphi$ at least until the time $t_{N}$ in Eq.(15.6), and the bound $\|\varphi(t)\| \leq R(t)$ with $R$ diverging at $t_{N}$; the result for this case can be applied to 
blow up problems, to get a lower bound on the time of explosion of the solution and an upper bound on its growth.

Example. We consider the Banach space

$$
\begin{gathered}
\mathbf{F}:=C_{0}(\mathbf{R}):=\{f: \mathbf{R} \rightarrow \mathbf{R} \mid f \text { is continuous, } f(x) \rightarrow 0 \text { for } x \rightarrow \infty\} ; \\
\|f\|:=\sup _{x \in \mathbf{R}}|f(x)| \quad \text { for } f \in \mathbf{F} .
\end{gathered}
$$

We define a linear semigroup $\mathcal{U}: t \in[0,+\infty) \rightarrow \mathcal{U}(t) \in \mathcal{L}(\mathbf{F})$ setting

$$
(\mathcal{U}(t) f)(x):=f(x+t)
$$

for all $f \in \mathbf{F} ; \mathcal{U}$ is strongly continuous, and $\|\mathcal{U}(t) f\|=\|f\|\left({ }^{3}\right)$. The generator of $\mathcal{U}$ is the operator

$$
\mathcal{A}:=\frac{d}{d x}: C_{0}^{1}(\mathbf{R}) \subset \mathbf{F} \rightarrow \mathbf{F}, \quad f \mapsto f_{x}
$$

where $C_{0}^{1}(\mathbf{R})$ is the space of the $C^{1}$ functions $f: \mathbf{R} \rightarrow \mathbf{R}$ such that $f, f_{x}$ vanish at infinity. We also introduce the function

$$
\mathcal{P}: \mathbf{F} \rightarrow \mathbf{F}, \quad \mathcal{P}(f):=f^{p} \quad(p>1 \text { integer }),
$$

which can be seen as a $t$-independent case of the Example on page 4, with $\mathcal{P}\left(f_{1}, \ldots, f_{p}\right):=$ $f_{1} \ldots f_{p} ;$ of course $\left\|\mathcal{P}\left(f_{1}, \ldots, f_{p}\right)\right\| \leq\left\|f_{1}\right\| \ldots\left\|f_{p}\right\|$.

We consider the Volterra problem (2.21) with $t_{0}:=0$, and an arbitrary initial datum $f_{0} \in \mathbf{F}$; in the special case $f_{0} \in C_{0}^{1}(\mathbf{R})$, the corresponding Cauchy problem is

$$
\dot{\varphi}(t)=\varphi(t)_{x}+\varphi(t)^{p}, \quad \varphi(0)=f_{0},
$$

involving a first order wave equation with polynomial nonlinearity. The results of the last Prop 5.2 can be applied in this framework with $U=1, B=0$ and $P=1$. For any $f_{0} \in \mathbf{F}$, this Proposition ensures existence of the solution $\varphi$ from time 0 to

$$
t_{N}:=\frac{1}{(p-1)\left\|f_{0}\right\|^{p-1}},
$$

(intending $t_{N}:=+\infty$ if $f_{0}=0$ ), and gives the bound

$$
\|\varphi(t)\| \leq R(t), \quad R(t):=\frac{\left\|f_{0}\right\|}{\left[1-(p-1)\left\|f_{0}\right\|^{p-1} t\right]^{\frac{1}{p-1}}}
$$

for all $t \in\left[0, t_{N}\right)$. In this case, the accuracy of the estimates in Prop 5.2 can be checked in a very direct way, because the maximal solution of (2.21) is known; this is given by

$$
\varphi(t)(x)=\frac{f_{0}(x+t)}{\left[1-(p-1) f_{0}(x+t)^{p-1} t\right]^{\frac{1}{p-1}}} \quad \text { for } t \in[0, \vartheta),
$$

\footnotetext{
${ }^{3}$ In fact, $\mathcal{U}$ can be extended to a linear group, also defined for $t \leq 0$, but we do not emphasize this aspect: our general framework is designed for time evolution in the future.
} 


$$
\vartheta:=\sup \left\{t>0 \mid(p-1) \sup _{x \in \mathbf{R}} f_{0}(x)^{p-1} t<1\right\} .
$$

If $f_{0} \in C_{0}^{1}(\mathbf{R})$, the above $\varphi$ also fulfils the Cauchy problem (5.15): this implies the full equivalence of the Volterra and Cauchy problems for such an $f_{0}$, in spite of the fact that item ii) of Prop 2.7 does not apply to the non reflexive Banach space $C_{0}(\mathbf{R})$. The following facts occur:

i) for $p$ odd, or $p$ even and $\sup _{x} f_{0}(x)=\sup _{x}\left|f_{0}(x)\right|, \vartheta$ equals the time $t_{N}$ in Eq.(5.16); thus, Prop 5.2 gives the best possible lower bound on the existence time of the maximal solution.

ii) For $p$ even and $0<\sup _{x} f_{0}(x)<\sup _{x}\left|f_{0}(x)\right|$, it is $+\infty>\vartheta>t_{N}$.

iii) For $p$ even and $\sup _{x} f_{0}(x) \leq 0<\sup _{x}\left|f_{0}(x)\right|$, it is $+\infty=\vartheta>t_{N}$.

The accuracy of the growth estimate (5.17) is easily analysed by comparison with Eq.(5.18). We think that better results would arise in cases ii) iii) by suitably generalizing the theory of approximate solutions to the framework of ordered Banach spaces [18; this will be done elsewhere.

\section{Approximate solutions on finite-dimensional submanifolds of $\mathbf{F}$.}

Let us discuss a general scheme to construct accurate approximate solutions, and apply to it Prop 3.4 to get information on the exact solution; a typical realization of this scheme is the Galerkin method, discussed in the sequel.

The framework. From now on: $\mathcal{U}$ is a strongly continuous linear semigroup with generator $\mathcal{A}$ and an estimator $u(t):=U e^{-B t}(U \geq 1, B \in \mathbf{R}) ; \mathcal{P}: \operatorname{Dom} \mathcal{P} \subset \mathbf{F} \times \mathbf{R} \rightarrow \mathbf{F}$ is continuous and Lipschitz on the strict subsets of its open domain; $\left(f_{0}, t_{0}\right) \in \operatorname{Dom} \mathcal{P}$.

Our idea is to construct an approximate solution $\varphi_{a p}$ for the Volterra problem (2.21), lying on a finite-dimensional (linear or nonlinear) submanifold of $\mathbf{F}$; we assume the latter to be coordinatized by some real parameters $a^{k}$, labelled by a finite set of indices $I$. More precisely, we consider an injective $C^{1}$ map

$$
\mathcal{G}: D o m \mathcal{G} \subset \mathbf{R}^{I} \rightarrow \mathbf{F}, \quad a=\left(a^{k}\right)_{k \in I} \mapsto \mathcal{G}(a),
$$

with open domain, such that the partial derivatives

$$
\partial_{k} \mathcal{G}(a) \equiv \frac{\partial \mathcal{G}}{\partial a^{k}}(a) \in \mathbf{F} \quad(k \in I)
$$

are linearly independent for all $a \in D o m \mathcal{G}$; we regard $I m \mathcal{G}$ as a multidimensional surface in $\mathbf{F}$. We also suppose that

$$
I m \mathcal{G} \subset D o m \mathcal{A}, \quad \operatorname{Im\mathcal {G}} \times\left[t_{0}, T\right) \subset \operatorname{Dom\mathcal {P}}
$$

for some $T \in\left(t_{0},+\infty\right]$, and ask $\mathcal{G}$ to be continuous as a map to Dom $\mathcal{A}$ with the graph norm. The approximate solution we consider has the form

$$
\varphi_{a p}(t):=\mathcal{G}(a(t)), \quad a() \in C^{1}\left(\left[t_{0}, t_{1}\right), D o m \mathcal{G}\right), \quad t \mapsto a(t)
$$


where $\left[t_{0}, t_{1}\right) \subset\left[t_{0}, T\right)$, and $a()$ is a function determined in the sequel. Clearly, the datum and differential errors of $\varphi_{a p}$ are

$$
\begin{gathered}
d\left(\varphi_{a p}\right):=\mathcal{G}\left(a\left(t_{0}\right)\right)-f_{0}, \\
e\left(\varphi_{a p}\right)(t):=\partial_{k} \mathcal{G}(a(t)) \dot{a}^{k}(t)-\mathcal{A} \mathcal{G}(a(t))-\mathcal{P}(\mathcal{G}(a(t)), t)
\end{gathered}
$$

(here and in the sequel, we employ the familiar Einstein's summation convention on repeated indices). We prescribe $a($ ) to fulfil a Cauchy problem

$$
\dot{a}(t)=X(a(t), t), \quad a\left(t_{0}\right)=a_{0}
$$

where $a_{0}$ is an initial datum, and $X: D o m \mathcal{G} \subset \mathbf{R}^{I} \rightarrow \mathbf{R}^{I}$ a continuous vector field; the criteria to fix $a_{0}$ and $X$ are discussed later.

For convenience, for all $a \in D o m \mathcal{G}, \dot{a} \in \mathbf{R}^{I}, t \in\left[t_{0}, T\right)$ we put

$$
\begin{gathered}
\hat{\delta}(a):=\left\|\mathcal{G}(a)-f_{0}\right\|, \quad \hat{\epsilon}(a, \dot{a}, t):=\left\|\mathcal{A G}(a)+\mathcal{P}(\mathcal{G}(a), t)-\partial_{k} \mathcal{G}(a) \dot{a}^{k}\right\| \\
\hat{\epsilon}(a, t):=\hat{\epsilon}(a, X(a, t), t)
\end{gathered}
$$

then, the approximate solution $\varphi_{a p}$ admits the datum and differential error estimators

$$
\delta:=\hat{\delta}\left(a_{0}\right), \quad \epsilon(t):=\hat{\epsilon}(a(t), t) .
$$

To conclude, we assume there are $\rho \in(0,+\infty]$ and a continuous function

$$
\hat{\ell}:[0, \rho) \times D o m \mathcal{G} \times\left[t_{0}, T\right) \rightarrow[0,+\infty), \quad(r, a, t) \mapsto \hat{\ell}(r, a, t),
$$

non decreasing in the variable $r$, such that $a \in D o m \mathcal{G}, t \in\left[t_{0}, T\right)$ and $\|f-\mathcal{G}(a)\|<\rho$ $\operatorname{imply}(f, t) \in \operatorname{Dom} \mathcal{P}$ and

$$
\|\mathcal{P}(f, t)-\mathcal{P}(\mathcal{G}(a), t)\| \leq \hat{\ell}(\|f-\mathcal{G}(a)\|, a, t) .
$$

Then, the function

$$
\ell(r, t):=\hat{\ell}(r, a(t), t)
$$

is a growth estimator for $\mathcal{P}$ from $\varphi_{a p}$. The application of Prop.s 3.4 3.6 gives

6.1 Proposition. Consider the equations

$$
\begin{gathered}
\dot{a}(t)=X(a(t), t), \quad a\left(t_{0}\right)=a_{0}, \\
\dot{R}(t)=U \hat{\epsilon}(a(t), t)+U \hat{\ell}(R(t), a(t), t)-B R(t), \quad R\left(t_{0}\right)=U \hat{\delta}\left(a_{0}\right),
\end{gathered}
$$

for the unknowns $a() \in C^{1}\left(\left[t_{0}, t_{1}\right)\right.$, Dom $\left.\mathcal{G}\right), R \in C^{1}\left(\left[t_{0}, t_{1}\right),[0, \rho)\right)$. If $(a(), R)$ is a solution on some interval $\left[t_{0}, t_{1}\right)$ and $\varphi_{a p}(t):=\mathcal{G}(a(t))$, then the Volterra problem (2.21) has a solution $\varphi$ on $\left[t_{0}, t_{1}\right)$, and $\left\|\varphi(t)-\varphi_{a p}(t)\right\| \leq R(t)$ on the same interval. 
Let us pass to the criteria for choosing $X$ and $a_{0}$. One of the most familiar is the Galerkin criterion (see, e.g., 4] or [15]): we will concentrate on it and will not discuss other approaches (such as the variational methods often used for the Lagrangian or Hamiltonian evolution equations, see, e.g., [6]). The Galerkin choice for $a_{0}$ and $X$ is the one minimizing the norms of the datum error and of the differential error (at any time):

6.2 Definition. The vector field $X$ and the datum $a_{0}$ fulfil the Galerkin criterion if

$$
\begin{gathered}
\hat{\epsilon}(a, \dot{a}, t)=\min ! \quad \text { for } \dot{a}=X(a, t), \\
\hat{\delta}(a)=\min ! \quad \text { for } a=a_{0}
\end{gathered}
$$

(the symbol min! indicating the absolute minimum).

(Of course, condition (6.16) is trivially satisfied if $f_{0}=\mathcal{G}\left(a_{0}\right)$; then the absolute minimum of $\hat{\delta}$, attained at this point, is zero).

Both equations (6.15) (6.16) can be studied in a systematic way if $\mathbf{F}$ is a Hilbert space, say real, with an inner product $<\mid>$ yielding the norm $\|f\|:=\sqrt{\langle f \mid f\rangle}$; from now on we stick to this assumption.

For all $a \in D o m \mathcal{G}$ we introduce the matrix

$$
\mathfrak{g}_{k l}(a):=<\partial_{k} \mathcal{G}(a) \mid \partial_{l} \mathcal{G}(a)>\quad(k, l \in I),
$$

which is symmetric and positive defined (recall the linear independence of the vectors $\left.\partial_{k} \mathcal{G}(a)\right)$. As customary in tensor calculus, we denote the inverse matrix with $\mathfrak{g}^{k h}(a)$ and introduce the convention of "raising and lowering indices" with these matrices. In connection with this, it is worthy to write down the identities

$$
\begin{gathered}
<\partial^{k} \mathcal{G}(a) \mid \partial_{h} \mathcal{G}(a)>=\delta^{k}{ }_{h} ; \\
v^{k}(a) \partial_{k} \mathcal{G}(a)=v_{k}(a) \partial^{k} \mathcal{G}(a), \\
<v^{k}(a) \partial_{k} \mathcal{G}(a) \mid w^{h}(a) \partial_{h} \mathcal{G}(a)>=v_{k}(a) w^{k}(a)=v^{k}(a) w_{k}(a) .
\end{gathered}
$$

Here: $\partial^{k} G(a):=\mathfrak{g}^{k h}(a) \partial_{h} G(a) \in \mathbf{F} ; v^{k}(a)(k \in I)$ is a family of real numbers, $v_{k}(a):=$ $\mathfrak{g}_{k h}(a) v^{h}(a)$ and $w^{k}(a), w_{k}(a)$ have a similar meaning. We apply all these notations to the discussion of the minimum problems (6.15), (6.16); the solutions are given by the two forthcoming Propositions.

6.3 Proposition. For any fixed $(a, t) \in D o m \mathcal{G} \times\left[t_{0}, T\right)$, the function $\dot{a} \mapsto \hat{\epsilon}^{2}(a, \dot{a}, t)$ is quadratic; it has a unique point of absolute minimum at

$$
\dot{a}^{k}=X^{k}(a, t), \quad X^{k}(a, t):=<\partial^{k} \mathcal{G}(a) \mid \mathcal{A G}(a)+\mathcal{P}(\mathcal{G}(a), t)>.
$$

The absolute minimum $\hat{\epsilon}(a, X(a, t), t):=\hat{\epsilon}(a, t)$ is given by

$$
\begin{gathered}
\hat{\epsilon}(a, t)^{2}=\|\mathcal{A G}(a)\|^{2}-<\mathcal{A} \mathcal{G}(a)\left|\partial_{k} \mathcal{G}(a)><\partial^{k} \mathcal{G}(a)\right| \mathcal{A} \mathcal{G}(a)>+ \\
+2<\mathcal{A G}(a)|\mathcal{P}(\mathcal{G}(a), t)>-2<\mathcal{A G}(a)| \partial_{k} \mathcal{G}(a)><\partial^{k} \mathcal{G}(a) \mid \mathcal{P}(\mathcal{G}(a), t)>+ \\
+\|\mathcal{P}(\mathcal{G}(a), t)\|^{2}-<\mathcal{P}(\mathcal{G}(a), t)\left|\partial_{k} \mathcal{G}(a)><\partial^{k} \mathcal{G}(a)\right| \mathcal{P}(\mathcal{G}(a), t)>.
\end{gathered}
$$


Proof. The general theory of Hilbert spaces tells us that, given a vector $g$ and a closed vector subspace $\mathcal{T}$ of $\mathbf{F}$, the problem

$$
\text { find } f \text { in } \mathcal{T} \text { such that }\|g-f\|=\min !
$$

has the unique solution

$$
f=\Pi g ;\|g-f\|^{2}=\|g\|^{2}-<g \mid \Pi g>, \Pi:=\text { the orthogonal projection of } \mathbf{F} \rightarrow \mathcal{T} .
$$

For any fixed $(a, t)$, the problem we have in mind has just the form (6.22): in this case

$$
\left.\mathcal{T}=\mathcal{T}(a):=\left\{\dot{a}^{k} \partial_{k} \mathcal{G}(a)\right) \mid \dot{a} \in \mathbf{R}^{I}\right\} \subset \mathbf{F},
$$

(which represents the tangent subspace at $\mathcal{G}(a)$ of $\operatorname{Im\mathcal {G}}$ ), the unknown $f$ is written $X^{k}(a, t) \partial_{k} \mathcal{G}(a)$ and

$$
g=\mathcal{A G}(a)+\mathcal{P}(\mathcal{G}(a), t), \quad \Pi=\Pi(a)=<\partial^{k} \mathcal{G}(a) \mid \cdot>\partial_{k} \mathcal{G}(a) ;
$$

computing from (6.23) the solution $f$ and the quantity $\|g-f\|^{2}=\hat{\epsilon}(a, t)^{2}$, we obtain Eq.s (6.20) 6.21).

\subsection{Proposition. Assume that:}

i) $\mathcal{G}$ is $C^{2}$, Dom $\mathcal{G}$ is convex;

ii) the matrix $\mathfrak{g}_{k l}(a)+<\mathcal{G}(a)-f_{0} \mid \partial_{k l}^{2} \mathcal{G}(a)>$ is semipositive for all $a \in$ DomG (where $\partial_{k l}^{2}$ are the second partial derivatives w.r.t. $\left.a^{k}, a^{l}\right)$;

iii) there is a point $a_{0}$ such that

$$
<\partial^{k} \mathcal{G}\left(a_{0}\right)\left|\mathcal{G}\left(a_{0}\right)>=<\partial^{k} \mathcal{G}\left(a_{0}\right)\right| f_{0}>.
$$

Then, the absolute minimum of $\hat{\delta}(a)$ is attained at $a=a_{0}$.

Proof. Everything follows computing the first and second derivatives of the function $a \rightarrow \hat{\delta}^{2}(a)$ in Eq.(6.7). Eq. (6.26) is the vanishing condition for the first derivatives, the other assumptions ensure that the stationary point $a_{0}$ is of absolute minimum.

Remark. The geometrical meaning of Eq.(6.26) is $\Pi\left(a_{0}\right) \mathcal{G}\left(a_{0}\right)=\Pi\left(a_{0}\right) f_{0}$, where $\Pi\left(a_{0}\right)$ is the orthogonal projection onto $\mathcal{T}\left(a_{0}\right)$, see (6.25).

The classical Galerkin method. All the previous formulas become very simple in the "classical" realization, where

$$
\mathcal{G}: \mathbf{R}^{I} \rightarrow \mathbf{F}, \quad a \mapsto \mathcal{G}(a)=a^{k} e_{k},
$$

$\left(e_{k}\right)_{k \in I}$ linearly independent vectors of $\operatorname{Dom\mathcal {A}}, \quad\left(e_{k}, t\right) \in D$ om $\mathcal{P}$ for $k \in I, t \in\left[t_{0}, T\right)$.

In this case $\operatorname{Im\mathcal {G}}$ is a vector subspace, and we have the identities

$$
\partial_{k} \mathcal{G}(a)=\text { const. }=e_{k}, \quad \mathfrak{g}_{k l}(a)=\text { const. }:=\mathfrak{g}_{k l}, \quad \partial^{k} \mathcal{G}(a)=\text { const. }=\mathfrak{g}^{k h} e_{h}:=e^{k} .
$$

(Also, the tangent space at any point $\mathcal{G}(a)$ is $\mathcal{T}(a)=$ const. $=\operatorname{Im} \mathcal{G}$; further simplifications occur in the orthonormal case where $\left.\mathfrak{g}_{k l}=\delta_{k l}, e^{k}=e_{k}\right)$. 
6.5 Proposition. The absolute minimum point for $\hat{\delta}$ and its value at this point are given by

$$
a_{0}^{k}=<e^{k} \mid f_{0}>, \quad \hat{\delta}\left(a_{0}\right)=\left\|f_{0}-a_{0}^{k} e_{k}\right\| .
$$

(In particular, $\hat{\delta}\left(a_{0}\right)=0$ if $f_{0}$ is in the linear subspace spanned by the family $\left(e_{k}\right)$ ).

Proof. Elementary (recall Eq.(6.26) ).

6.6 Proposition. Suppose $\mathcal{P}$ to be determined by a p-linear map $\mathcal{P}$, as in the Example of page 4 . Then, Eq.s (6.20) and (6.21) become

$$
\begin{gathered}
X^{k}(a, t)=<e^{k}\left|\mathcal{A} e_{l}>a^{l}+<e^{k}\right| \mathcal{P}\left(e_{l_{1}}, \ldots, e_{l_{p}}, t\right)>a^{l_{1}} \ldots a^{l_{p}} \\
\hat{\epsilon}(a, t)^{2}=\left(<\mathcal{A} e_{j}\left|\mathcal{A} e_{l}>-<\mathcal{A} e_{j}\right| e_{k}><e^{k} \mid \mathcal{A} e_{l}>\right) a^{j} a^{l}+ \\
+2\left(<\mathcal{A} e_{j}\left|\mathcal{P}\left(e_{l_{1}}, \ldots, e_{l_{p}}, t\right)>-<\mathcal{A} e_{j}\right| e_{k}><e^{k} \mid \mathcal{P}\left(e_{l_{1}}, \ldots, e_{l_{p}}, t\right)>\right) a^{j} a^{l_{1}} \ldots a^{l_{p}}+ \\
+\left(<\mathcal{P}\left(e_{j_{1}}, \ldots, e_{j_{p}}, t\right) \mid \mathcal{P}\left(e_{l_{1}}, \ldots, e_{l_{p}}, t\right)>+\right. \\
\left.-<\mathcal{P}\left(e_{j_{1}}, \ldots, e_{j_{p}}, t\right)\left|e_{k}><e^{k}\right| \mathcal{P}\left(e_{l_{1}}, \ldots, e_{l_{p}}, t\right)>\right) a^{j_{1}} \ldots a^{j_{p}} a^{l_{1}} \ldots a^{l_{p}}
\end{gathered}
$$

In the r.h.s. of the last equation, the coefficients of $a^{j} a^{l}$ and $a^{j} a^{l_{1}} \ldots a^{l_{p}}$ are zero if the subspace spanned by the family $\left(e_{k}\right)$ is invariant under $\mathcal{A}$ (which occurs, in particular, if each $e_{k}$ is an eigenvector of $\left.\mathcal{A}\right)$.

Proof. Both Eq.s 66.30 6.31) follow easily from $\partial_{k} \mathcal{G}(a)=e_{k}$ and from the multilinearity of $\mathcal{P}$. In the second equation the coefficients of $a^{j} a^{l}$ and $a^{j} a^{l_{1}} \ldots a^{l_{p}}$ are, respectively,

$$
\begin{gathered}
<\mathcal{A} e_{j}\left|\mathcal{A} e_{l}>-<\mathcal{A} e_{j}\right| e_{k}><e^{k}\left|\mathcal{A} e_{l}>=<\mathcal{A} e_{j}\right| \mathcal{A} e_{l}>-<\mathcal{A} e_{j} \mid \Pi \mathcal{A} e_{l}> \\
<\mathcal{A} e_{j}\left|\mathcal{P}\left(e_{l_{1}}, \ldots, e_{l_{p}}, t\right)>-<\mathcal{A} e_{j}\right| e_{k}><e^{k} \mid \mathcal{P}\left(e_{l_{1}}, \ldots, e_{l_{p}}, t\right)>= \\
=<\mathcal{A} e_{j}\left|\mathcal{P}\left(e_{l_{1}}, \ldots, e_{l_{p}}, t\right)>-<\Pi \mathcal{A} e_{j}\right| \mathcal{P}\left(e_{l_{1}}, \ldots, e_{l_{p}}, t\right)>
\end{gathered}
$$

where $\Pi$ is the projection on the linear subspace spanned by $\left(e_{k}\right)$. If this subspace is left invariant by $\mathcal{A}$ we have $\Pi \mathcal{A} e_{l}=\mathcal{A} e_{l}$ for each $l \in I$, so the above coefficients are zero. $\diamond$

6.7 Proposition. Assume again $\mathcal{P}$ to be as in the Example of page 4 . Then, the growth of the function $\mathcal{P}$ starting from a point $\mathcal{G}(a)=a^{k} e_{k}$ admits this estimate, for all $f \in \boldsymbol{F}$ and $t \in \Delta$ :

$$
\begin{gathered}
\|\mathcal{P}(f, t)-\mathcal{P}(\mathcal{G}(a), t)\| \leq \hat{\ell}(\|f-\mathcal{G}(a)\|, a, t) \\
\hat{\ell}:[0,+\infty) \times \boldsymbol{R}^{I} \times \Delta \rightarrow[0,+\infty), \quad \hat{\ell}(r, a, t):=P(t) \sum_{j=1}^{p}\left(\begin{array}{c}
p \\
j
\end{array}\right){\sqrt{a_{k} a^{k}}}^{p-j} r^{j} .
\end{gathered}
$$


Proof. Apply Eq.(2.17) with $f^{\prime}=\mathcal{G}(a)$ and $t^{\prime}=t$; note that $\left\|f^{\prime}\right\|=\sqrt{a_{k} a^{k}}$.

In applications of the classical Galerkin method, some choices for the family $\left(e_{k}\right)$ have a special consideration. Apart from systems of eigenvectors of $\mathcal{A}$, other choices occur in finite elements methods, which are strictly related to the idea of approximating the evolutionary problem by space discretization; in this case, $\left(e_{k}\right)$ is typically a family of piecewise linear (or polynomial) "chapeau functions" related to some spatial grid, see e.g. [4].

\section{Applications to the nonlinear heat equation.}

Our aim is to discuss the nonlinear heat equation $\dot{f}=f_{x x}+f^{p}$ with Dirichlet boundary conditions, for $x$ ranging in $(0, \pi)$ (we work in one space dimension only for simplicity). Let us introduce this equation in the framework of Sobolev spaces.

Notations for Sobolev spaces. All functions on $(0, \pi)$ are real-valued; $\mathcal{F}(0, \pi)$ means $\mathcal{F}((0, \pi), \mathbf{R})$ for each functional class $\mathcal{F}$. We consider the Hilbert space $L^{2}(0, \pi)$, with the standard inner product $\langle f \mid g\rangle_{L^{2}}:=\int_{0}^{\pi} d x f(x) g(x)$; here the functions

$$
s_{k}(x):=\sqrt{\frac{2}{\pi}} \sin (k x) \quad(k \in\{1,2,3, \ldots\})
$$

form a complete orthonormal system. We introduce the Sobolev space

$$
H^{1}(0, \pi):=\left\{f \in L^{2}(0, \pi) \mid f_{x} \in L^{2}(0, \pi)\right\} \subset C([0, \pi])
$$

$f_{x}$ denoting the distributional derivative of $f$; this is a Hilbert space with the inner product

$$
<f|g>:=<f| g>_{L^{2}}+<f_{x} \mid g_{x}>_{L^{2}}
$$

yielding the norm $\|f\|:=\sqrt{\langle f \mid f\rangle}$. The inclusion indicated in (7.2) is well known, and allows to define $f(0), f(\pi)$ for all $f \in H^{1}(0, \pi)$; we fix the attention on the closed subspace

$$
\mathbf{F}:=H_{0}^{1}(0, \pi):=\left\{f \in H^{1}(0, \pi) \mid f(0)=f(\pi)=0\right\}
$$

and equip it with the restriction of the inner product (7.3). It turns out that

$$
\mathbf{F}=\left\{f \in L^{2}(0, \pi)\left|\sum_{k=1}^{\infty} k^{2}<s_{k}\right| f>_{L^{2}}^{2}<+\infty\right\}
$$

The functions $s_{k}$ form a complete orthogonal system for this space: it is

$$
\begin{gathered}
<s_{k}\left|s_{l}>=\left(1+k^{2}\right) \delta_{k l}, \quad<s_{k}\right| f>=\left(1+k^{2}\right)<s_{k} \mid f>_{L^{2}} \quad \forall f \in \mathbf{F}, \\
<f\left|g>=\sum_{k=1}^{\infty}\left(1+k^{2}\right)<f\right| s_{k}>_{L^{2}}<s_{k} \mid g>_{L^{2}} \quad \forall f, g \in \mathbf{F} .
\end{gathered}
$$


Both $H^{1}(0, \pi)$ and $\mathbf{F}$ are known to be Banach algebras with respect to the pointwise product. We will use systematically the inequality (almost optimal, see the Appendix A)

$$
\|f g\| \leq\|f\|\|g\| \quad \forall f, g \in \mathbf{F} .
$$

The operator $\mathcal{A}$. This is the linear map

$$
\mathcal{A}:=\frac{d^{2}}{d x^{2}} \text { on } \operatorname{Dom} \mathcal{A}:=\left\{f \in \mathbf{F} \mid f_{x x} \in \mathbf{F}\right\} .
$$

Of course, for all $k$,

$$
\mathcal{A} s_{k}=-k^{2} s_{k} .
$$

The operator $\mathcal{A}$ generates the strongly continuous linear semigroup $\mathcal{U}$ on $\mathbf{F}$, defined by

$$
\mathcal{U}(t) f:=\sum_{k=1}^{\infty} e^{-k^{2} t}<s_{k} \mid f>_{L^{2}} s_{k} \quad \text { for } t \in[0,+\infty), f \in \mathbf{F}
$$

the above series is in fact convergent in $\mathbf{F}$, and

$$
\|\mathcal{U}(t) f\|=\sqrt{\sum_{k=1}^{\infty} e^{-2 k^{2} t}\left(1+k^{2}\right)<s_{k} \mid f>_{L^{2}}^{2}} \leq e^{-t}\|f\| .
$$

The nonlinear function $\mathcal{P}$. This is defined by

$$
\mathcal{P}: \mathbf{F} \rightarrow \mathbf{F}, \quad \mathcal{P}(f):=f^{p} \quad(p>1 \text { integer }) .
$$

It belongs to the class of maps in the Example of page 4 and corresponds to the time independent $p$-linear map

$$
\mathcal{P}: \times^{p} \mathbf{F} \rightarrow \mathbf{F}, \quad \mathcal{P}\left(f_{1}, \ldots, f_{p}\right):=f_{1} \ldots f_{p} .
$$

Of course, Eq.(17.7) implies $\left\|\mathcal{P}\left(f_{1}, \ldots, f_{p}\right)\right\| \leq\left\|f_{1}\right\| \ldots\left\|f_{p}\right\|$.

The Volterra and Cauchy problems. We consider the Volterra problem (2.21) on $\mathbf{F}$, with $\mathcal{U}$ as before, $\mathcal{P}(f, t):=$ the above defined $\mathcal{P}(f), t_{0}:=0$ and some initial datum $f_{0} \in \mathbf{F}$; this reads

$$
\varphi(t)=\mathcal{U}(t) f_{0}+\int_{0}^{t} d s \mathcal{U}(t-s) \varphi(s)^{p} .
$$

From now on, we will denote with

$$
\varphi:[0, \vartheta) \rightarrow \mathbf{F}
$$

the maximal solution. If $f_{0} \in D o m \mathcal{A},(2.21)$ is fully equivalent to the Cauchy problem

$$
\dot{\varphi}(t)=\varphi(t)_{x x}+\varphi(t)^{p}, \quad \varphi(0)=f_{0}
$$


(because $\mathbf{F}$ is reflexive and $\mathcal{P}$ Lipschitz on the strict subsets of its domain).

Kaplan's blow up criterion. We specialize to the present framework a general citerion of Kaplan [5] for the blow up in a finite time of the solution of a nonlinear parabolic equation. To this purpose, we introduce the function

$$
Q: L^{2}(0, \pi) \rightarrow \mathbf{R} \quad f \mapsto Q(f):=\frac{1}{2}<\sin \mid f>_{L^{2}}=\frac{1}{2} \int_{0}^{\pi} d x \sin x f(x) .
$$

7.1 Proposition. Consider the Volterra problem 17.14; if

$$
f_{0} \geq 0, \quad Q\left(f_{0}\right)>1
$$

then

$$
\vartheta \leq t_{K}, \quad t_{K}:=-\frac{1}{(p-1)} \log \left(1-\frac{1}{Q\left(f_{0}\right)^{p-1}}\right) .
$$

Proof. It is sketched in the Appendix B] adapting Kaplan's general argument.

From now on, $t_{K}$ will be referred to as the Kaplan time for the datum $f_{0}$.

Basic estimates on the solution. As a first step in our analysis, let us apply Prop.s 5.1 and 5.2 to the Volterra problem. In the case we are considering, the semigroup has an estimator $u(t)=U e^{-B t}$ with $U=1, B=1$; also, it is $\|\mathcal{P}(f)\| \leq \ell(\|f\|)$ with $\ell(r):=r^{p}$. Therefore, we have

7.2 Proposition. For any initial datum $f_{0} \in \boldsymbol{F}$, it is $\vartheta \geq t_{N}$ and $\|\varphi(t)\| \leq R(t)$ for all $t \in\left[0, t_{N}\right)$, with $t_{N}$ and $R$ depending on the norm of $f_{0}$ in the following way:

$$
\begin{gathered}
t_{N}:= \begin{cases}+\infty & \text { if }\left\|f_{0}\right\| \leq 1, \\
-\frac{1}{(p-1)} \log \left(1-\frac{1}{\left\|f_{0}\right\|^{p-1}}\right) & \text { if }\left\|f_{0}\right\|>1,\end{cases} \\
R(t):=\frac{\left\|f_{0}\right\|}{\left[1-\left(\left\|f_{0}\right\|^{p-1}-1\right)\left(e^{(p-1) t}-1\right)\right]^{\frac{1}{p-1}}} .
\end{gathered}
$$

If $\left\|f_{0}\right\|<1, R$ is decreasing and $R(t) \rightarrow 0$ for $t \rightarrow+\infty$. If $\left\|f_{0}\right\|=1, R(t)=1$ for all $t$. If $\left\|f_{0}\right\|>1, R$ is increasing and $R(t) \rightarrow+\infty$ for $t \rightarrow t_{N}^{-}$.

Summary of the previous results on $\vartheta$. An example. We have

$$
t_{N} \leq \vartheta \text { for all } f_{0} \in \mathbf{F} ; \quad \vartheta \leq t_{K} \quad \text { if } f_{0} \geq 0, Q\left(f_{0}\right)>1,
$$

with $t_{N}$ as in (7.20), $t_{K}$ as in (7.19). Let us consider, in particular, the initial datum

$$
f_{0}(x):=A s_{1}(x)=\sqrt{\frac{2}{\pi}} A \sin x, \quad(A \geq 0) ;
$$


then $f_{0} \in D o m \mathcal{A}$, so we have a full equivalence of (7.14) with the Cauchy problem (7.16). It turns out that

$$
\left\|f_{0}\right\|=\frac{A}{C_{N}}, C_{N}:=\frac{\sqrt{2}}{2}=0.7071 . . \quad ; \quad Q\left(f_{0}\right)=\frac{A}{C_{K}}, C_{K}:=2 \sqrt{\frac{2}{\pi}}=1.595 . .
$$

Therefore, Eq.(17.22) with this choice of the datum tells us that

$$
\begin{aligned}
\vartheta=+\infty \text { if } 0 \leq A \leq C_{N} ; t_{N} \leq \vartheta \text { if } C_{N}<A \leq C_{K} ; t_{N} \leq \vartheta \leq t_{K} \text { if } A>C_{K}, \\
t_{N}:=-\frac{1}{p-1} \log \left(1-\frac{C_{N}^{p-1}}{A^{p-1}}\right) \sim_{A \rightarrow+\infty} \frac{1}{p-1} \frac{C_{N}^{p-1}}{A^{p-1}}, \\
t_{K}:=-\frac{1}{p-1} \log \left(1-\frac{C_{K}^{p-1}}{A^{p-1}}\right) \sim_{A \rightarrow+\infty} \frac{1}{p-1} \frac{C_{K}^{p-1}}{A^{p-1}} .
\end{aligned}
$$

It should be noted that (7.22) does not allow to establish whether $\vartheta$ is finite or infinite, for $A$ in the interval $\left(C_{N}, C_{K}\right]$. In the rest of the Section, we will infer more precise estimates about $\vartheta$ by means of the Galerkin method, and also rediscuss its behaviour for large $A$.

A Galerkin approach to the nonlinear heat equation. We apply the scheme of Sect 6 with

$$
I \text { a finite subset of }\{1,2,3, \ldots\}, \quad \mathcal{G}(a):=a^{k} s_{k} \text { for all } a=\left(a^{k}\right) \in \mathbf{R}^{I}
$$

and $s_{k}$ the functions (7.1). We refer, in particular, to the description given in the previous Section for the "classical" Galerkin method, to be employed with $e_{k}:=s_{k},<\mid>$ the inner product (7.3) on $\mathbf{F}:=H_{0}^{1}(0, \pi)$, and

$$
\mathfrak{g}_{k l}=\left(1+k^{2}\right) \delta_{k l}, \quad \mathfrak{g}^{k l}=\frac{1}{1+k^{2}} \delta^{k l}
$$

these matrices are used to raise and lower indices. The vector field $X$, the error function $\hat{\epsilon}$ and the growth estimator function $\hat{\ell}$ of Eq.s (6.30) (6.31) 6.34) are time independent, and given by

$$
\begin{gathered}
X^{k}(a):=-k^{2} a^{k}+<s^{k} \mid s_{l_{1}} \ldots s_{l_{p}}>a^{l_{1}} \ldots a^{l_{p}} \\
\hat{\epsilon}(a)^{2}:=\left(<s_{j_{1}} \ldots s_{j_{p}}\left|s_{l_{1}} \ldots s_{l_{p}}>-<s_{j_{1}} \ldots s_{j_{p}}\right| s_{k}><s^{k} \mid s_{l_{1}} \ldots s_{l_{p}}>\right) a^{j_{1}} \ldots a^{j_{p}} a^{l_{1}} \ldots a^{l_{p}}, \\
\hat{\ell}(r, a):=\sum_{j=1}^{p}\left(\begin{array}{c}
p \\
j
\end{array}\right) \sqrt{a_{k} a^{k}{ }^{p-j} r^{j}}
\end{gathered}
$$

(see the observation following Eq.(17.13); also, note that $a_{k} a^{k}=\sum_{k \in I}\left(1+k^{2}\right)\left(a^{k}\right)^{2}$ ). This amount of information (completed by Eq. (6.29) for the initial datum) must be inserted into the general scheme of Prop 6.1 the solution of the finite dimensional system (6.13), (6.14) appearing therein provides simultaneously:

i) a pair of functions $a(), R()$ on an interval $\left[0, t_{G}\right)$, the former giving the approximate solution $\varphi_{a p}(t):=a^{k}(t) s_{k}$. In the sequel $t_{G}$ will be called the Galerkin time; 
ii) an assurance that the Volterra problem (7.14) has a maximal solution $\varphi$ on $[0, \theta) \supset$ $\left[0, t_{G}\right)$, and a bound $\left\|\varphi(t)-\varphi_{a p}(t)\right\| \leq R(t)$ for all $t<t_{G}$.

Introducing an example. We assume

$$
p:=2, \quad f_{0} \text { as in (7.23) . }
$$

Problem (7.14 7.16) will be treated with a "two-modes" application of the Galerkin method; more precisely, we will work on the linear submanifold spanned by $\left(s_{k}\right)_{k \in I}$, setting

$$
I:=\{1,3\}, \quad \alpha:=a^{1}, \quad \gamma:=a^{3} ;
$$

(the choice $I=\{1,2,3\}$ would not yield any improvement, because the function $t \mapsto a^{2}(t)$ would be ultimately found to be zero). The vector field, the error function and the growth function of Eq.s (7.30) (7.31) (77.32) are given by

$$
\begin{gathered}
X^{\alpha}(\alpha, \gamma)=-\alpha+\sqrt{\frac{2}{\pi^{3}}}\left(\frac{8}{3} \alpha^{2}-\frac{16}{15} \alpha \gamma+\frac{72}{35} \gamma^{2}\right) \\
X^{\gamma}(\alpha, \gamma):=-9 \gamma+\sqrt{\frac{2}{\pi^{3}}}\left(-\frac{8}{15} \alpha^{2}+\frac{144}{35} \alpha \gamma+\frac{8}{9} \gamma^{2}\right) ; \\
\hat{\epsilon}(\alpha, \gamma)^{2}=\left(\frac{7}{2 \pi}-\frac{512}{15 \pi^{3}}\right) \alpha^{4}+\left(\frac{34816}{315 \pi^{3}}-\frac{10}{\pi}\right) \alpha^{3} \gamma+ \\
+\left(\frac{46}{\pi}-\frac{12172288}{33075 \pi^{3}}\right) \alpha^{2} \gamma^{2}-\frac{22528}{175 \pi^{3}} \alpha \gamma^{3}+\left(\frac{39}{2 \pi}-\frac{3247616}{99225 \pi^{3}}\right) \gamma^{4} \\
\hat{\ell}(\alpha, \gamma, r)=r^{2}+2 \sqrt{2 \alpha^{2}+10 \gamma^{2}} r .
\end{gathered}
$$

According to (6.29), the initial conditions for $\alpha(t)$ and $\gamma(t)$ are, respectively,

$$
<s^{1}\left|f_{0}>=A, \quad<s^{3}\right| f_{0}>=0 ;
$$

the corresponding datum error is zero. In conclusion, we have to study the system

$$
\begin{gathered}
\dot{\alpha}=X^{\alpha}(\alpha, \gamma), \quad \dot{\gamma}=X^{\gamma}(\alpha, \gamma), \quad \dot{R}=\hat{\epsilon}(\alpha, \gamma)+\hat{\ell}(\alpha, \gamma, R)-R, \\
\alpha(0)=A, \quad \gamma(0)=0, \quad R(0)=0,
\end{gathered}
$$

for the unknown functions $t \mapsto \alpha(t), \gamma(t), R(t)$. This cannot be solved analytically, but can be easily treated by any package for the numerical solution of ordinary differential equations; an integration algorithm with adaptative control of the step size gives an excellent approximation for the solution of (17.39), also including the evaluation of its existence time. All statements that follow about the system (7.39) are based on the MATHEMATICA package; thus, expression such as "the solution of (7.39)", etc., always indicate the MATHEMATICA output (of which we report the first digits).

New estimates on the existence time $\vartheta$. We have the bounds

$$
t_{G} \leq \vartheta \text { for all } A \geq 0, \quad \vartheta \leq t_{K} \text { for } A>C_{K}
$$


involving the Galerkin and Kaplan times, defined previously (the latter depends on $C_{K}=$ 1.595..). It is found that

$$
\begin{gathered}
t_{G}=+\infty \quad \text { for } 0 \leq A \leq \mathcal{C}_{G}, \quad \mathcal{C}_{G}=1.056 . . \\
\alpha(t), \gamma(t), R(t) \rightarrow 0 \quad \text { for } t \rightarrow+\infty \text { and } \quad 0 \leq A \leq \mathcal{C}_{G} .
\end{gathered}
$$

It should be noted that the bound $\mathcal{C}_{G}$ on $A$ for the global existence of (17.16) is better than the previously derived bound $C_{N}=0.7071 \ldots$ For larger values of $A$, the existence time $t_{G}$ for (7.39) is finite. The forthcoming Table reports $t_{G}$ for some values of $A$ above the Kaplan critical value $C_{K}$, as well as the corresponding values of $t_{K}$. It also reports

$$
\eta:=\frac{t_{K}-t_{G}}{t_{K}+t_{G}}
$$

which is the relative uncertainty about the actual existence time $\vartheta$ of (7.16) corresponding to the lower and upper bounds $t_{G}, t_{K}$.

\begin{tabular}{|l|l|l|l|}
\hline$A$ & $t_{G}$ & $t_{K}$ & $\eta$ \\
\hline$\leq 1.056 .$. & $+\infty$ & & \\
1.60 & $1.104 .$. & $5.935 .$. & $0.6861 .$. \\
2 & $0.7730 .$. & $1.598 .$. & $0.3481 .$. \\
4 & $0.3138 .$. & $0.5090 .$. & $0.2372 .$. \\
10 & $0.1112 .$. & $0.1738 .$. & $0.2196 .$. \\
20 & $0.05340 .$. & $0.08315 .$. & $0.2177 .$. \\
\hline
\end{tabular}

The $A \rightarrow+\infty$ limit for the previous estimates is easily discussed. To determine the asymptotics of $t_{G}$, we reexpress the unknown functions $\alpha(t), \gamma(t)$ and $R(t)$ in terms of three rescaled functions $\mathrm{t} \rightarrow \mathrm{a}(\mathrm{t}), \mathrm{c}(\mathrm{t}), \mathrm{R}(\mathrm{t})$, depending on $\mathrm{t}:=A t$ and defined by

$$
\alpha(t)=A \mathrm{a}(A t), \quad \gamma(t)=A \mathrm{c}(A t), \quad R(t)=A \mathrm{R}(A t) .
$$

Then, the system (7.39) becomes (with ${ }^{\prime}:=d / d \mathrm{t}$ )

$$
\begin{gathered}
\mathrm{a}^{\prime}=-\frac{\mathrm{a}}{A}+\sqrt{\frac{2}{\pi^{3}}}\left(\frac{8}{3} \mathrm{a}^{2}-\frac{16}{15} \mathrm{ac}+\frac{72}{35} \mathrm{c}^{2}\right), \mathrm{c}^{\prime}=-\frac{9 \mathrm{c}}{A}+\sqrt{\frac{2}{\pi^{3}}}\left(-\frac{8}{15} \mathrm{a}^{2}+\frac{144}{35} \mathrm{ac}+\frac{8}{9} \mathrm{c}^{2}\right), \\
\mathrm{R}^{\prime}=\hat{\epsilon}(\mathrm{a}, \mathrm{c})+\hat{\ell}(\mathrm{a}, \mathrm{c}, \mathrm{R})-\frac{\mathrm{R}}{A} \\
\mathrm{a}(0)=1, \quad \mathrm{c}(0)=0, \quad \mathrm{R}(0)=0 .
\end{gathered}
$$

In the $A \rightarrow+\infty$ limit, the terms a/ $A, \mathrm{c} / A$ and $\mathrm{R} / A$ can be neglected and the outcoming system (7.46) $)_{\infty}$ is $A$-independent. The numerical treatment of this limit system shows that the solution $\mathrm{t} \rightarrow \mathrm{a}(\mathrm{t}), \mathrm{c}(\mathrm{t}), \mathrm{R}(\mathrm{t})$ exists for $\mathrm{t} \in\left[0, C_{G}\right)$, where

$$
C_{G}=1.026 .
$$


Returning to the standard time $t=\mathrm{t} / A$, we conclude that

$$
t_{G} \sim \frac{C_{G}}{A} \text { for } A \rightarrow+\infty .
$$

It should be noted that all values of $A$ in the previous Table are seen empirically to fulfil the inequality

$$
t_{G} \geq-\frac{C_{G}}{\mathfrak{C}_{G}} \log \left(1-\frac{\mathfrak{C}_{G}}{A}\right),
$$

with $t_{G}$ very close to the r.h.s. To compare these results with the Kaplan upper bound recall that, for all $A>C_{K}=1.596 .$. ,

$$
t_{K}=-\log \left(1-\frac{C_{K}}{A}\right) \sim_{A \rightarrow+\infty} \frac{C_{K}}{A} .
$$

Due to (7.48) (7.50), the relative uncertainty (7.43) has the limit

$$
\eta \rightarrow \frac{C_{K}-C_{G}}{C_{K}+C_{G}}=0.2173 . . \quad \text { for } A \rightarrow+\infty .
$$

As a matter of fact, in this limit case one can find directly the asymptotics for the actual existence time $\vartheta$ of (17.14)(7.16). In fact, if one writes the maximal solution $\varphi$ as

$$
\varphi(t)=A \chi(A t)
$$

one obtains for $\chi$ the Cauchy problem

$$
\chi^{\prime}(\mathrm{t})=\frac{1}{A} \chi(\mathrm{t})_{x x}+\chi^{2}(\mathrm{t}), \quad \chi(0)(x)=\sqrt{\frac{2}{\pi}} \sin x .
$$

For $A \rightarrow+\infty$, the differential equation becomes $\chi^{\prime}(\mathrm{t})=\chi^{2}(\mathrm{t})$, and the solution is

$$
\chi(\mathrm{t})(x)=\frac{\sqrt{2 / \pi} \sin (x)}{1-\sqrt{2 / \pi} \mathrm{t} \sin x} \quad \text { for } \mathrm{t} \in[0, \sqrt{\pi / 2}) ;
$$

so, returning to the standard time $t=\mathrm{t} / A$, we conclude

$$
\vartheta \sim_{A \rightarrow+\infty} \frac{\sqrt{\pi / 2}}{A}=\frac{1.253 . .}{A} .
$$

The constant $\sqrt{\pi / 2}$ is fairly close to the arithmetic mean of the costants $C_{G}$ and $C_{K}$, which appear in the asymptotic expressions (7.48), (7.50) for the lower and upper bounds $t_{G}, t_{K}$. Thus, the actual existence time $\vartheta$ should be close to the arithmetic mean of $t_{G}$ and $t_{K}$ if $A$ is sufficiently large. An attack to the Cauchy problem (7.16) that we performed approximating $d^{2} / d x^{2}$ by finite differences seems to indicate that this actually occurs for all $A \gtrsim 2$. 
Analysis of the Galerkin solution. After solving the system (7.39) for a given $A$, one constructs the corresponding approximate solution for (7.16) such that, for $t \in\left[0, t_{G}\right)$,

$$
\varphi_{a p}(t)(x)=\alpha(t) \sqrt{\frac{2}{\pi}} \sin x+\gamma(t) \sqrt{\frac{2}{\pi}} \sin (3 x) ; \quad\left\|\varphi_{a p}(t)\right\|=\sqrt{2 \alpha(t)^{2}+10 \gamma(t)^{2}} .
$$

The system (7.39) also gives a function $R$ such that $\left\|\varphi_{a p}(t)-\varphi(t)\right\| \leq R(t)$ for $t$ in the same interval; let us illustrate the behaviour of the above functions for two values of $A$.

Case $\boldsymbol{A}=1$. It is $t_{G}=+\infty$, which implies $\vartheta=+\infty$. Figures 1 2 , 4 give the graphs of the functions $t \mapsto \alpha(t), \gamma(t),\left\|\varphi_{a p}(t)\right\|$ and $R(t)$, all converging to zero for $t \rightarrow+\infty$. Figure 3 gives the function $x \in(0, \pi) \mapsto \varphi_{a p}(t)(x)$ at three fixed times. For the exact solution $\varphi$ of (7.16), we infer

$$
\|\varphi(t)\| \leq\left\|\varphi_{a p}(t)\right\|+R(t) \rightarrow 0 \quad \text { for } t \rightarrow+\infty .
$$

Figure 5 is a graph of the relative bound $R(t) /\left\|\varphi_{a p}(t)\right\|$ in a time interval where it is fairly little.

Case $\boldsymbol{A}=4$. The Galerkin system (7.39) has a finite existence time $t_{G}=0.3138 \ldots$ Figures 6] 10 give information of the same kind as the figures of the case $A=1$, but describe a qualitatively different behaviour; in particular, the function $t \mapsto R(t)$ diverges for $t \rightarrow t_{G}$. 


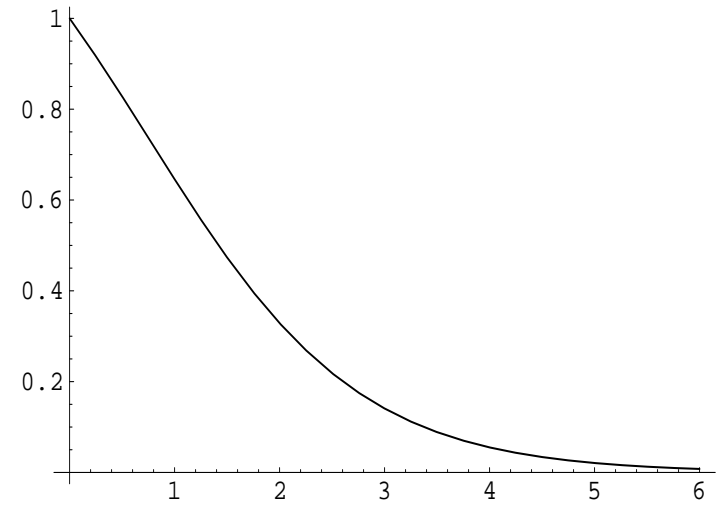

Figure 1: $A=1$. Graph of $\alpha(t)$.

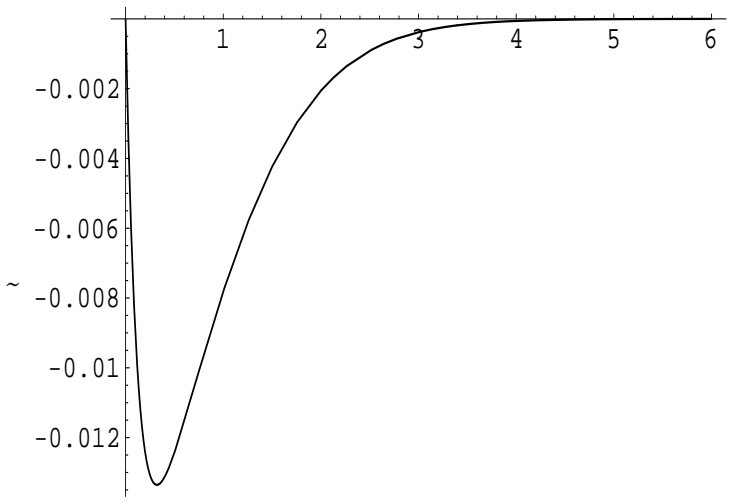

Figure 2: $A=1$. Graph of $\gamma(t)$.

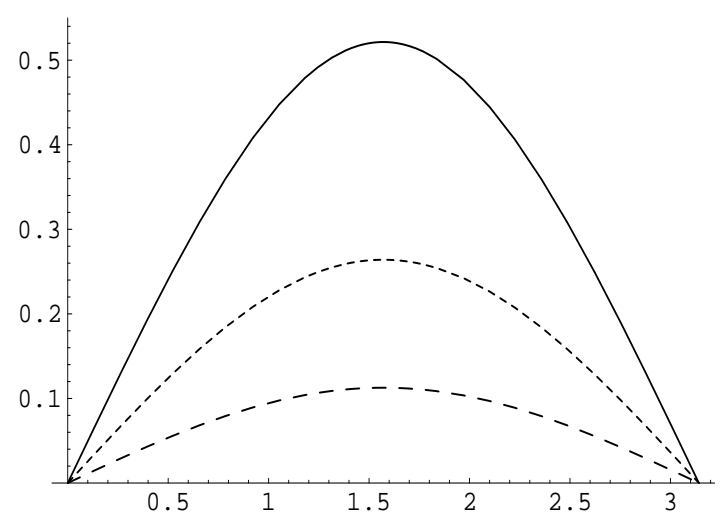

Figure 3: $A=1$. Graphs of $\varphi_{a p}(t)(x)$ for $x \in(0, \pi)$ and $t=1$ (continuous line), $t=2$ (short dashes), $t=3$ (long dashes).

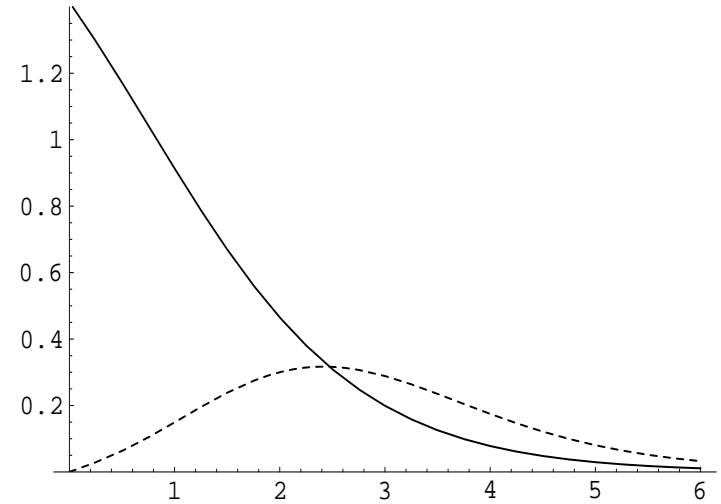

Figure 4: $A=1$. Graphs of $\left\|\varphi_{a p}(t)\right\|$ (continuous line) and $R(t)$ (dashed line).

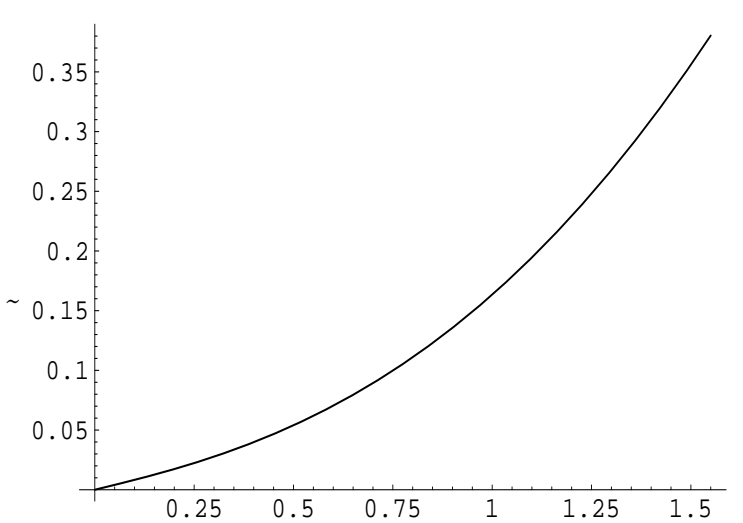

Figure 5: $A=1$. Graph of $R(t) /\left\|\varphi_{a p}(t)\right\|$. 


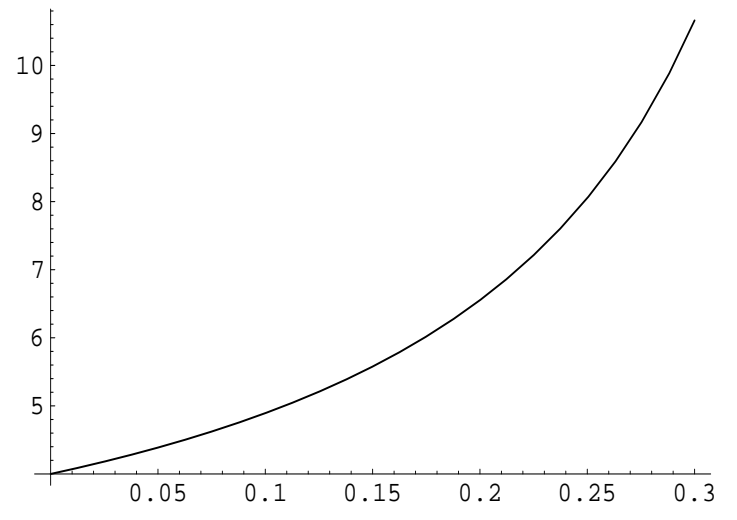

Figure 6: $A=4$. Graph of $\alpha(t)$.

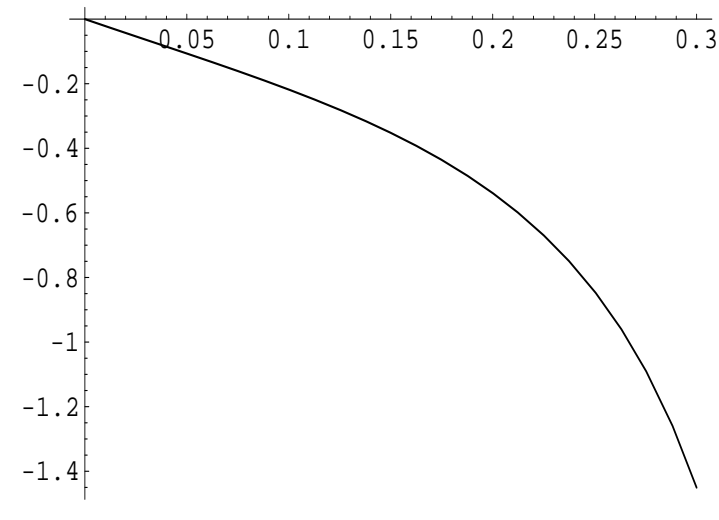

Figure 7: $A=4$. Graph of $\gamma(t)$.

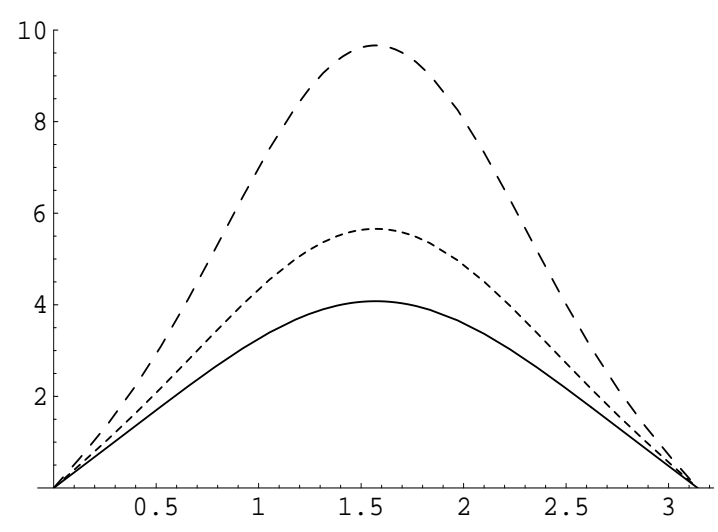

Figure 8: $A=4$. Graphs of $\varphi_{a p}(t)(x)$ for $x \in(0, \pi)$ and $t=0.1$ (continuous line), $t=0.2$ (short dashes), $t=0.3$ (long dashes).
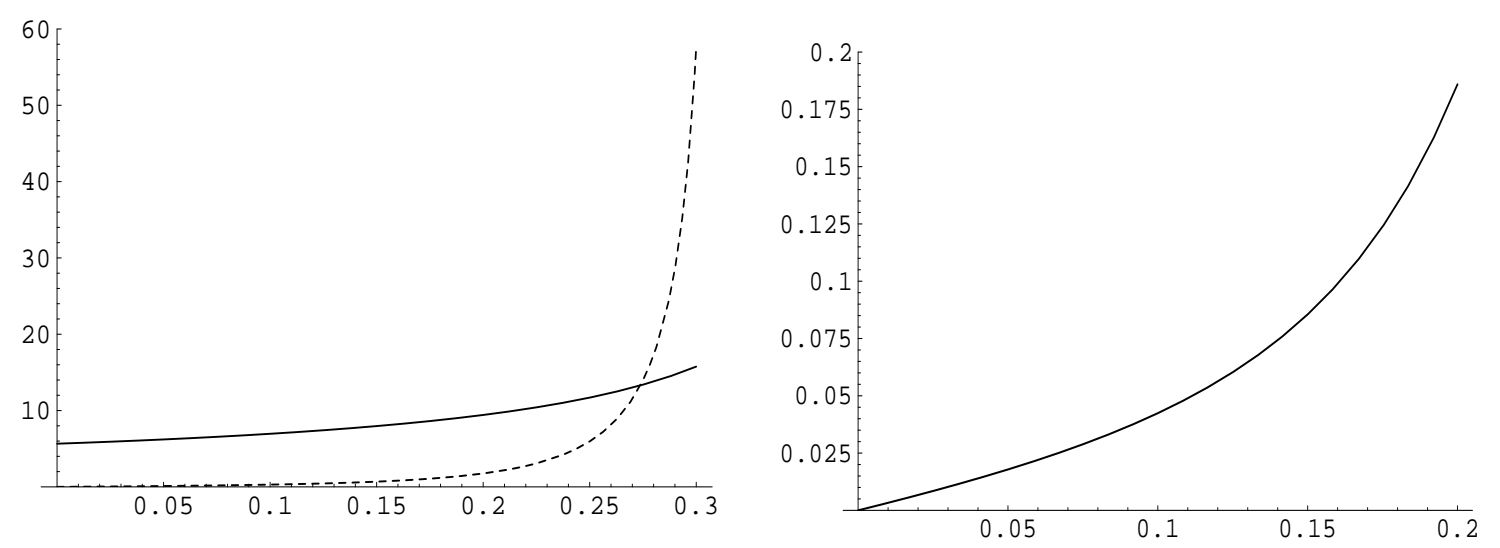

Figure 9: $A=4$. Graphs of $\left\|\varphi_{a p}(t)\right\|$ (continuous line) and $R(t)$ (dashed line).

Figure 10: $A=4$. Graph of $R(t) /\left\|\varphi_{a p}(t)\right\|$. 


\section{A Appendix. Sobolev spaces and pointwise product.}

We consider the space $H^{1}(\mathbf{R}, \mathbf{C}):=\left\{f: \mathbf{R} \rightarrow \mathbf{C} \mid f, f_{x} \in L^{2}(\mathbf{R})\right\}$ with the inner product $<f|g>:=<f| g>_{L^{2}}+<f_{x} \mid g_{x}>_{L^{2}}$ and the corresponding norm \|\| . This is useful to treat the space $H_{0}^{1}(0, \pi)$ of Sect 7 (made of real functions): in fact, there is an $\mathbf{R}$-linear, norm preserving inclusion

$$
H_{0}^{1}(0, \pi) \subset H^{1}(\mathbf{R}, \mathbf{C}),
$$

where each $f \in H_{0}^{1}(0, \pi)$ is extended to the full real axis setting $f(x):=0$ for $x \notin(0, \pi)$. Both $H_{0}^{1}(0, \pi)$ and $H^{1}(\mathbf{R}, \mathbf{C})$ are closed under the pointwise product, and $\|f g\| \leq$ const. $\|f\|\|g\|$ for all functions therein [14]. We claim the following:

A.1 Proposition. Consider the sharp (i.e., the minimum) constants $L, M$ in the inequalities

$$
\begin{aligned}
\|f g\| \leq L\|f\|\|g\| & \text { for all } f, g \in H_{0}^{1}(0, \pi) ; \\
\|f g\| \leq M\|f\|\|g\| & \text { for all } f, g \in H^{1}(\boldsymbol{R}, \boldsymbol{C}) .
\end{aligned}
$$

Then

$$
0.811<L \leq M \leq 1 .
$$

Proof. i) $L \leq M$ follows readily from (A.1).

ii) A lower bound for $L$ follows applying (A.2) with $f=g=f_{\lambda}$, where

$$
f_{\lambda}(x):=e^{-\lambda|x-\pi / 2|}-e^{-\lambda \pi / 2} \quad(\lambda>0),
$$

The norms $\left\|f_{\lambda}\right\|,\left\|f_{\lambda}^{2}\right\|$ are computed in an elementary way, and we get a minorant of $L$ for each $\lambda$. The best lower bound is attained for $\lambda$ close to 1.55, and implies $L>0.811$. iii) Let us prove that $M \leq 1$. To this purpose, we employ for the complex functions $f$ on $\mathbf{R}$ the Fourier transform $(\mathcal{F} f)(k)=\frac{1}{\sqrt{2 \pi}} \int \mathbf{R} d x e^{-i k x} f(x)$; this yields the representation

$$
H^{1}(\mathbf{R}, \mathbf{C})=\left\{\left.f \in L^{2}(\mathbf{R}, \mathbf{C})\left|+\infty>\int_{\mathbf{R}} d k\left(1+k^{2}\right)\right| \mathcal{F} f(k)\right|^{2}=\|f\|^{2}\right\},
$$

and sends pointwise product into $(1 / \sqrt{2 \pi}) \times$ the convolution product $*$. Consider any two functions $f, g \in H^{1}(\mathbf{R}, \mathbf{C})$. Then the following holds:

$$
\begin{gathered}
\|f g\|^{2}=\int_{\mathbf{R}} d k\left(1+k^{2}\right)|\mathcal{F}(f g)(k)|^{2}=\frac{1}{2 \pi} \int_{\mathbf{R}} d k\left(1+k^{2}\right)|(\mathcal{F} f * \mathcal{F} g)(k)|^{2} ; \\
(\mathcal{F} f * \mathcal{F} g)(k)=\int_{\mathbf{R}} d h \mathcal{F} f(k-h) \mathcal{F} g(h)= \\
=\int_{\mathbf{R}} d h \frac{1}{\sqrt{1+(k-h)^{2}} \sqrt{1+h^{2}}}\left(\sqrt{1+(k-h)^{2}} \mathcal{F} f(k-h) \sqrt{1+h^{2}} \mathcal{F} g(h)\right) ; \\
\frac{1}{2 \pi}|(\mathcal{F} f * \mathcal{F} g)(k)|^{2} \leq C(k) P(k),
\end{gathered}
$$




$$
\begin{gathered}
C(k):=\frac{1}{2 \pi} \int_{\mathbf{R}} \frac{d h}{\left(1+(k-h)^{2}\right)\left(1+h^{2}\right)}=\frac{1}{4+k^{2}}, \\
P(k):=\int_{\mathbf{R}} d h\left(1+(k-h)^{2}\right)|\mathcal{F} f(k-h)|^{2}\left(1+h^{2}\right)|\mathcal{F} g(h)|^{2} .
\end{gathered}
$$

Eq.(A.9) follows from Hölder's inequality $\left|\int d h U V\right|^{2} \leq\left(\int d h|U|^{2}\right)\left(\int d h|V|^{2}\right)$; inserting (A.9) into Eq.(A.7), we get

$$
\begin{aligned}
\|f g\|^{2} \leq \int_{\mathbf{R}} d k\left(1+k^{2}\right) C(k) P(k) & \leq\left(\sup _{k \in \mathbf{R}}\left(1+k^{2}\right) C(k)\right) \int_{\mathbf{R}} d k P(k)= \\
= & 1 \times\|f\|^{2}\|g\|^{2} .
\end{aligned}
$$

In [1] we have discussed the constants for more general inequalities related to the pointwise product and to the spaces $H^{n}\left(\mathbf{R}^{d}, \mathbf{C}\right)$. The upper bound $M \leq 1$ derived now improves the result arising from [1] in the special case of the inequality (A.3); the method employed here to bind $M$ develops in a fully quantitative way an idea suggested in [13].

\section{B Appendix. Proof of Prop.7.1.}

We keep all notations of Sect 7 The proof consists of the following steps:

i) The function $Q$ of Eq.(17.17) can be seen as a continuous linear form, both on $L^{2}(0, \pi)$ and on $\mathbf{F}$. For all $f \in \mathbf{F}$ and $t \in[0,+\infty)$, we easily infer from (7.10) that

$$
Q(\mathcal{U}(t) f)=e^{-t} Q(f) .
$$

ii) Let $f \in L^{2 p}(0, \pi)\left(\subset L^{2}(0, \pi)\right)$ and $f \geq 0$; then

$$
Q(f) \leq Q\left(f^{p}\right)^{1 / p}
$$

This follows taking $q$ such that $1 / p+1 / q=1$, and writing $Q(f)=\int_{0}^{\pi} d x u(x) v(x)$ with $u(x):=\left(\frac{1}{2} \sin x\right)^{1 / q}, v(x):=\left(\frac{1}{2} \sin x\right)^{1 / p} f(x)$; Hölder's inequality $\int u v \leq\left(\int u^{q}\right)^{1 / q}\left(\int v^{p}\right)^{1 / p}$ yields Eq.(B.2) $\left({ }^{4}\right)$.

iii) We consider the maximal solution $\varphi:[0, \theta) \rightarrow \mathbf{F}$ of the Volterra problem (7.14) with datum $f_{0} \in \mathbf{F}$, assuming $f_{0} \geq 0$ and $Q\left(f_{0}\right)>1$; the nonnegativity of $f_{0}$ implies $\varphi(t) \geq 0$ for all $t$ (see, e.g., [2]). We define the (continuous) function

$$
t \in[0, \theta) \mapsto Q(t):=Q(\varphi(t))
$$

and note that

$$
Q(t) \geq 0, \quad Q(t) \geq e^{-t} Q\left(f_{0}\right)+\int_{0}^{t} d s e^{-(t-s)} Q(s)^{p}
$$

\footnotetext{
${ }^{4}$ Eq.(B.2) is optimal, in this sense: the best constant in the inequality $Q(f) \leq C Q\left(f^{p}\right)^{1 / p}$ for all nonnegative $L^{2 p}$ functions is $C=1$. This is true even if we restrict the inequality to much smaller classes, such as the nonnegative $C^{\infty}$, compactly supported functions $f$ on $(0, \pi)$.
} 
the first bound follows from $\varphi(t) \geq 0$, and the second one from (7.14) (B.1) (B.2).

iv) For each $n \in \mathbf{N}$, we define a continuous function $S_{n}:[0, \vartheta) \rightarrow \mathbf{R}$ by

$$
S_{0}(t):=e^{-t} Q\left(f_{0}\right), \quad S_{n+1}(t):=e^{-t} Q\left(f_{0}\right)+\int_{0}^{t} d s e^{-(t-s)} S_{n}(s)^{p}
$$

it is proved recursively that, for all $n \in \mathbf{N}$ and $t \in[0, \vartheta)$,

$$
Q(t) \geq S_{n}(t) \geq 0
$$

(the first inequality depends on (B.4), the second one is elementary). It is easily checked that the sequence of functions $\left(S_{n}\right)$ is a Cauchy sequence in the topology of the uniform convergence on all compact subintervals $[0, \tau] \subset[0, \vartheta)$; its $n \rightarrow+\infty$ limit is a continuous function $S:[0, \vartheta) \rightarrow \mathbf{R}$ such that, for all $t$ in this interval,

$$
S(t)=e^{-t} Q\left(f_{0}\right)+\int_{0}^{t} d s e^{-(t-s)} S(s)^{p}, \quad Q(t) \geq S(t) \geq 0 .
$$

From the above integral equation, we see that $S$ is in fact $C^{1}$, and fulfils the Cauchy problem

$$
\dot{S}(t)=S(t)\left(S(t)^{p-1}-1\right), \quad S(0)=Q\left(f_{0}\right)
$$

this has a unique maximal solution (for nonnegative times), denoted again with $t \mapsto S(t)$, which extends the function considered up to now on $[0, \vartheta)$, and is given by

$$
\int_{Q\left(f_{0}\right)}^{S(t)} \frac{d r}{r\left(r^{p-1}-1\right)}=t \quad \text { for } t \in\left[0, t_{K}\right), \quad t_{K}:=\int_{Q\left(f_{0}\right)}^{+\infty} \frac{d r}{r\left(r^{p-1}-1\right)}
$$

furthermore, $S(t) \rightarrow+\infty$ for $t \rightarrow t_{K}^{-}$. Computing the last integral, we see that $t_{K}$ has the expression (7.19) in the statement of the theorem; we know that $\vartheta \leq t_{K}$, so the proof of Prop 7.1 is concluded.

Acknowledgments. We are grateful to the anonymous referees for some suggestions that stimulated an improvement of the paper. This work was partly supported by INdAM and by MIUR, COFIN 2001 Research Project "Geometry of Integrable Systems".

\section{References}

[1] C. Caratheodory, Calculus of variations and partial differential equations of the first order, Holden-Day, San Francisco (1965).

[2] T. Cazenave, A. Haraux, An introduction to semilinear evolution equations, Oxford Univ. Press, New York (1998).

[3] N. Dunford, J.T. Schwartz, Linear Operators. I, Interscience, New York (1958). 
[4] D.R. Durran, Numerical methods for wave equations in geophysical fluid dynamics, Springer, New York (1999).

[5] S. Kaplan, On the growth of solutions of quasi-linear parabolic equations, Commun. Pure Appl. Mathematics 16, 305-330 (1963).

[6] Y.B. Kivshar, B.A. Malomed, Dynamics of solitons in nearly integrable systems, Rev. Mod. Phys. 61, 763-915 (1989).

[7] V. Lakshmikantham, S. Leela, A.A. Martynyuk, Stability analysis of nonlinear systems, Marcel Dekker, New York (1989).

[8] P. Lochak, C. Meunier, Multiphase averaging for classical systems. With applications to adiabatic theorems, Appl. Math. Sciences 72, Springer-Verlag, New York (1988).

[9] E.N. Lorenz, Deterministic nonperiodic flow, J. Atmospheric Sci. 20 130-141 (1963).

[10] D.S. Mitrinovic, J.E. Pecaric, A.M. Fink, Inequalities involving functions and their integrals and derivatives, Kluwer, Dordrecht (1991).

[11] C. Morosi, L. Pizzocchero, On the constants in some inequalities for the Sobolev norms and pointwise product, J. of Inequal. \& Appl. 7, 421-452 (2002).

[12] C. Olech, On the existence and uniqueness of solutions of an ordinary differential equation in the case of Banach space, Bull. Acad. Polon. Sci. Sér. Sci. Math. Astron. Phys. 8, 668-673 (1960).

[13] J. Pöschel, Quasi-periodic solutions for a nonlinear wave equation, Comment. Math. Helvetici 71, 269-296 (1996).

[14] T. Runst, W. Sickel, Sobolev spaces of fractional order, Nemytskij operators and nonlinear partial differential equations, de Gruyter, Berlin (1996).

[15] R. Temam, Infinite-dimensional dynamical systems in mechanics and physics, Springer, New York (1988).

[16] W. Walter, Differential and integral inequalities, Springer, New York (1970)

[17] T. Wazewski, Sur l'existence et l' unicité des intégrales des équations différentielles ordinaires au cas de l'espace de Banach, Bull. Acad. Polon. Sci. Sér. Sci. Math. Astron. Phys. 8, 301-305 (1960).

[18] E. Zeidler, Non linear functional analysys and its applications I. Fixed-point theorems, Springer, New York (1986).

[19] E. Zeidler, Non linear functional analysys and its applications II/B. Nonlinear monotone operators, Springer, New York (1990). 